\title{
Cloud information content analysis of multi-angular measurements in the oxygen A-band: application to 3MI and MSPI
}

\author{
Guillaume Merlin $^{1}$, Jérôme Riedi $^{1}$, Laurent C. Labonnote ${ }^{1}$, Céline Cornet ${ }^{1}$, Anthony B. Davis ${ }^{2}$, Phillipe Dubuisson ${ }^{1}$, \\ Marine Desmons ${ }^{1}$, Nicolas Ferlay ${ }^{1}$, and Frédéric Parol ${ }^{1}$ \\ ${ }^{1}$ Laboratoire d'Optique Atmosphérique, Université de Lille 1, Sciences et Technologies, Villeneuve d'Ascq, France \\ ${ }^{2}$ Jet Propulsion Laboratory, California Institute of Technology, Pasadena, CA, USA
}

Correspondence to: Jérôme Riedi (jerome.riedi@univ-lille1.fr)

Received: 14 September 2015 - Published in Atmos. Meas. Tech. Discuss.: 4 December 2015

Revised: 30 July 2016 - Accepted: 3 August 2016 - Published: 11 October 2016

\begin{abstract}
Information content analyses on cloud top altitude (CTOP) and geometrical thickness (CGT) from multi-angular A-band measurements in the case of monolayer homogeneous clouds are conducted. In the framework of future multi-angular radiometer development, we compared the potential performances of the 3MI (Multiviewing, Multi-channel and Multi-polarization Imaging) instrument developed by EUMETSAT, which is an extension of POLDER/PARASOL instrument and MSPI (Multiangle SpectroPolarimetric Imager) developed by NASA's Jet Propulsion Laboratory. Quantitative information content estimates were realized for thin, moderately opaque and opaque clouds for different surface albedo and viewing geometry configurations. Analyses show that retrieval of CTOP is possible with a high accuracy in most of the cases investigated. Retrieval of CGT is also possible for optically thick clouds above a black surface, at least when CGT $>1-2 \mathrm{~km}$ and for thin clouds for CGT $>2-3 \mathrm{~km}$. However, for intermediate optical thicknesses $(\mathrm{COT} \simeq 4$ ), we show that the retrieval of CGT is not simultaneously possible with CTOP. A comparison between 3MI and MSPI shows a higher information content for MSPI's measurements, traceable to a thinner filter inside the oxygen A-band, yielding higher signal-tonoise ratio for absorption estimation. Cases of cloud scenes above bright surfaces are more complex but it is shown that the retrieval of CTOP remains possible in almost all situations while the information content on CGT appears to be insufficient in many cases, particularly for COT $<4$ and CGT $<2-3 \mathrm{~km}$.
\end{abstract}

\section{Introduction}

Clouds play an important role in the Earth radiation budget by modulating both the incoming and outgoing solar and thermal radiation that in turn directly act on the heating and cooling rates within the atmosphere. In particular, the cloud cover vertical distribution has a significant impact on a large number of meteorological and climatic processes. For example, Johansson et al. (2015) show that cloud vertical structure has a strong impact on the summer monsoon over the Indian subcontinent. Furthermore, cloud vertical extent plays a crucial role in the radiative budget of the Earth (Ohring and Adler, 1978) and this effect is still poorly understood, especially for low clouds (L'Ecuyer et al., 2009). By influencing the distribution of heating within the atmosphere, it drives the cloud dynamics and evolution of clouds' macroand micro-structure. All climate feedbacks linked to clouds are still poorly known (Stocker et al., 2013) and a better understanding of climate evolution has to go through a better description of the macrophysical cloud properties, such as cloud top and bottom altitudes.

Satellites are the most convenient tool for obtaining a complete picture of cloud macrophysics over a broad range of spatial and temporal scales and active sensors such as spaceborne radar (Mace et al., 2009) or lidar (Winker and Trepte, 1998; Winker et al., 2007) are particularly well suited to characterize their vertical structure. However, they can only provide information under a narrow swath, which hampers their ability to provide global coverage on a daily basis. 
In contrast, passive sensors allow for a larger coverage but, so far, the accuracy of retrieved cloud top altitude is limited and the possibility of obtaining cloud geometrical thickness has yet to be consolidated (Ferlay et al., 2010; Desmons et al., 2013). Numerous techniques have been developed to retrieve cloud height from passive sensors. The $\mathrm{CO}_{2}$-slicing method uses $\mathrm{CO}_{2}$ differential absorption in the thermal infrared spectral range (Rossow and Schiffer, 1991; King et al., 1992; Platnick et al., 2003). Ultraviolet radiances can also be used as rotational Raman scattering causes depletion or filling of solar Fraunhofer lines in the UV spectrum, depending on the Rayleigh scattering above the cloud (Joiner and Bhartia, 1995; de Beek et al., 2001). Similarly, the polarization of reflected light, at visible shorter wavelength, due to Rayleigh scattering carries information on cloud top height (Goloub et al., 1994; Knibbe et al., 2000). Finally, cloud top height can also be retrieved accurately thanks to geometrical methods applied to stereo observations (Moroney et al., 2002; Seiz et al., 2007; Wu et al., 2009).

Information on cloud top location from passive instruments can also be obtained through the exploitation of dioxygen differential absorption of solar radiation in the A-band, as first suggested by Yamamoto and Wark (1961). Indeed, molecular oxygen is a well-mixed atmospheric gas and, consequently, the amount of absorption is linked to the photon geometrical path length in the atmosphere, which is limited at a first order by the cloud top altitude. However, clouds are not solid (surface-like) reflectors. The additional absorption of radiation occurring along the path inside the cloud leads to significant biases and uncertainties in the retrieved cloud-top pressure, as shown by Preusker and Lindstrot (2009). Some studies have shown, however, that this multiple-scattering effect can be used to obtain information on photon path lengths within the clouds, which in turn could provide information on cloud geometrical thickness (e.g. Davis et al. (2009); Asano et al. (1995); Kuji and Nakajima (2002) and references therein). Depending on the instrument characteristics in terms of spectral resolution and coverage and multi-angular capabilities, different methods have been proposed or applied. Rozanov and Kokhanovsky (2004) developed a retrieval technique exploiting hyperspectral A-band measurements from SCIAMACHY, thanks to a semi-analytical algorithm using asymptotic theory. Previous information content analyses (Heidinger and Stephens, 2000; Schuessler et al., 2014) have explored the information content of high-resolution spectral measurements in the Aband (Heidinger and Stephens, 2000) and were applied to different sensors such as GOME and GOME-2 (Schuessler et al., 2014; Loyola et al., 2007). Our present study focuses on the $\mathrm{O}_{2}$ A-band information content arising from multiangle observations. Turning to narrowband bispectral multiangular measurements in the A-band, Ferlay et al. (2010) suggested using the correlation between the angular standard deviation of the cloud oxygen pressure and the cloud geometrical thickness. The feasibility of this technique was later on confirmed by Desmons et al. (2013), who compared POLDER3/PARASOL retrievals of cloud geometrical thickness to information derived from collocated active sensors (CALIOP/CLOUDSAT). At the same time, a similar approach proposed using the differential absorption occurring between single-view measurements in the $\mathrm{O}_{2} \mathrm{~A}$ - and B-bands (Yang et al., 2013).

Our work aims to develop a comprehensive analysis of the information provided by multi-angle and bispectral measurements in the $\mathrm{O}_{2}$ A-band to derive the vertical location and extent of clouds (cloud top altitude and cloud geometrical thickness). Building on previous analyses by Ferlay et al. (2010) and Desmons et al. (2013), our present analysis aims to understand the feasibility of retrieving those parameters for all types of clouds (liquid/ice, thin/thick) and for a range of surface albedo values. But our study remains limited to homogeneous single-layer clouds without aerosols. This investigation is motivated by the need to develop physics-based algorithms that will be applied globally to measurements from a new generation of multi-angle sensors.

Several studies have demonstrated the great interest of combining multi-spectral, multi-angular and polarized measurements in the visible, near and shortwave infrared to better constrain retrievals of cloud microphysical (Bréon and Doutriaux-Boucher, 2005; Zhang et al., 2009; Riedi et al., 2010) and macrophysical (Ferlay et al., 2010; Desmons et al., 2013) properties. In this context, several missions that will fly a new generation of polarimetric multi-angular sensors that are currently being formulated. Here, we focus on the Multiviewing, Multi-channel, Multi-polarization Imaging mission (3MI) on the EUMETSAT Polar System-Second generation (EPS-SG) and the Multiangle SpectroPolarimetric Imager (MSPI) proposed, among others, for NASA's AerosolCloud-Ecosystem (ACE) mission.

This paper is organized as follows. In Sect. 2, we describe the main characteristics of the $3 \mathrm{MI}$ and MSPI instruments that are relevant to our study and describe the simulation scenario performed for each. In Sect. 3, we qualitatively illustrate and discuss the sensitivity of multi-angle A-band measurements to cloud top altitude and geometrical thickness. In Sect. 4, we go further by developing a formal information content analysis of multi-angle $\mathrm{O}_{2}$ A-band measurements and discuss the impact of various model assumptions and errors on available information. In particular, the cases of dark and bright surfaces are discussed to evaluate the feasibility of a retrieval algorithm that could be applied globally. In Sect. 5, we discuss our results and potential pathways to counteract the limitations of multi-angular measurements in the A-band in order to obtain information on both cloud top altitude and geometrical thickness. The main results are summarized in the concluding section. 


\section{Observation assumptions and simulations}

The 3MI and MSPI sensors both combine multi-viewing, multi-wavelength measurements with polarization. Particularities of the two instrument characteristics are presented hereafter focusing primarily on their differences with respect to A-band measurements.

\subsection{MI characteristics}

The Multi-viewing, Multi-Channel, Multi-Polarization Imaging mission (3MI) is planned as part of the future generation of EUMETSAT polar satellites, EPS-SG. 3MI has been designed to observe Earth reflectance in a wide spectral range $(410-2130 \mathrm{~nm})$ under up to 14 different viewing directions with polarimetric capabilities in 9 out of a total of 12 spectral channels. The 3MI concept largely leverages three previous Polarization and Directionality of the Earth's Reflectances (POLDER) missions and some scientific limitations have been overcome by enhancing the instrument capabilities along all three dimensions. Specifically, the total spectral range has been extended to cover shorter (visible $410 \mathrm{~nm}$ ) and longer (shortwave infrared) wavelengths (Table 1), the spatial resolution has been increased to $4 \mathrm{~km} \times 4 \mathrm{~km}$ at nadir and the swath of the instrument will provide complete daily coverage of the Earth from an EPS orbit.

Regarding the A-band sampling, in addition to the absorbing channel centred on the A-band at $763 \mathrm{~nm}$ (in band), 3MI is anticipated to provide measurements in a broader $40 \mathrm{~nm}$ wide channel at $765 \mathrm{~nm}$ (broad-band) extending on both sides of the A-band similarly to what has been used for POLDER. As will be illustrated later on and following previous studies by Ramon et al. (2003), a narrow spectral channel at $754 \mathrm{~nm}$ (out-band) outside the A-band could provide higher information content when paired with the $763 \mathrm{~nm}$ channel. However, the broader $765 \mathrm{~nm}$ channel appears more appropriate for 3MI in terms of instrument design and stability over the mission, particularly due to the need for vicarious calibration of the 3MI. It remains that a $754 \mathrm{~nm}$ channel will also be available from the METImage instrument on EPS-SGm, providing an opportunity for synergistic use with $3 \mathrm{MI}$. Therefore, in addition to the anticipated 3MI baseline configuration $(763 / 765 \mathrm{~nm})$, we also studied the information provided by a potentially available $763 / 754 \mathrm{~nm}$ pair of measurements. These pairs of measurements are usually used in the form of ratios between the absorbing band in the A-band and a nonabsorbing band with approximatively the same scattering properties. We will employ then the narrowband/broadband A-band ratio (Eq. 1) for the $763+765 \mathrm{~nm}$ configuration and in/out A-band ratio (Eq. 2) for the $763+754 \mathrm{~nm}$ configuration.

$$
\begin{aligned}
& R_{\text {thin/broad }}=I_{763} / I_{765} \\
& R_{\text {in } / \text { out }}=I_{763} / I_{754}
\end{aligned}
$$

\begin{tabular}{|c|c|c|c|}
\hline & $\begin{array}{c}\text { Central } \\
\text { wavelength } \\
(\mathrm{nm})\end{array}$ & $\begin{array}{l}\text { Bandwidth } \\
\quad(\mathrm{nm})\end{array}$ & Polarization \\
\hline$B$ & 410 & 20 & $\mathrm{Y}$ \\
\hline \multirow{8}{*}{ 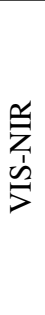 } & 443 & 20 & $\mathrm{Y}$ \\
\hline & 490 & 20 & Y \\
\hline & 555 & 20 & $\mathrm{Y}$ \\
\hline & 670 & 20 & $\mathrm{Y}$ \\
\hline & 763 & 10 & $\mathrm{~N}$ \\
\hline & 765 & 40 & $\mathrm{~N}$ \\
\hline & 865 & 40 & $\mathrm{Y}$ \\
\hline & 910 & 20 & Y \\
\hline \multirow{3}{*}{$\sum_{\infty}^{\infty}$} & 1370 & 40 & Y \\
\hline & 1650 & 40 & Y \\
\hline & 2130 & 40 & $\mathrm{Y}$ \\
\hline
\end{tabular}

Table 1. Future 3MI spectral bands.

\subsection{MSPI characteristics}

The Multiangle SpectroPolarimetric Imager, or MSPI, is a candidate for the multi-directional, multi-wavelength, highaccuracy polarization imager identified by the National Research Council's Earth Sciences Decadal Survey as one component of the national Aerosol-Cloud-Ecosystem, or ACE, mission. NASA would fly the ACE spacecraft late in the next decade to characterize the role of aerosols in climate forcing, especially their impact on precipitation and cloud formation. MSPI is conceptually similar to JPL's Multiangle Imaging SpectroRadiometer, or MISR, carried on NASA's EOS Terra spacecraft, but with some important additions. The new camera design extends the spectral range to the ultraviolet and shortwave infrared (from 446-866 to $355-2130 \mathrm{~nm}$ ), increasing the image swath (from 360 to $680 \mathrm{~km}$ ) to achieve more rapid global coverage (from 4 to 9 days), and adds highaccuracy polarimetry in selected spectral bands. Like MISR, a suite of MSPI cameras would view Earth at a variety of angles with an intrinsic pixel size of a few hundred metres, which for certain channels would be averaged up to about $1 \mathrm{~km}$.

At present, MSPI has ground-based (Diner et al., 2010) and airborne (Diner et al., 2013) versions with only VNIR (visible near infrared) spectral coverage. The latter, AirMSPI development is currently entering a new phase. In particular, AirMSPI-2 will have extended spectral coverage into the SWIR with polarization and additional A-band channels without polarization. MSPI will provide measurements in a absorbing channel centred in the oxygen A-band (in band) and a narrow spectral channel at $754 \mathrm{~nm}$ (out band). Similarly to 3MI, we used the following in/out A-band ratio for MSPI. 


\subsection{Simulation assumptions}

The use of observations in an absorption band, in essence, relies on the interpretation of average photon optical path length cumulated within the atmosphere, and especially above the cloud, when trying to retrieve cloud top pressure or altitude (denoted in the following as CTOP). This path length is estimated through differential absorption occurring between two channels in and around the $\mathrm{O}_{2}$ A-band, providing an estimate of the atmospheric transmission above cloud.

Historically, the ratio of measurements in two nearby channels was used to estimate this atmospheric $\mathrm{O}_{2}$ A-band transmission and two approaches have been used from past sensors to establish this ratio. The first one uses a ratio between a radiance in the A-band and a radiance completely outside so that it is not affected at all by absorption but is close enough to consider other atmospheric and surface properties as constant. This configuration has been used successfully for instance with Medium Resolution Imaging Spectrometer (MERIS), although an accurate spectral calibration was identified as critical (Ramon et al., 2003). The other one uses a broader band covering the A-band (thin band/broadband) entirely, which again provides similar atmospheric and surface properties in both channels. It presents the advantage of being less sensitive to calibration issues at the expenses of a lower signal to noise (because the broadband signal is partly affected by absorption; therefore it provides less contrast with respect to the absorption channel). This configuration has been used for the three previous POLDER missions and proved to be a robust solution when instrument calibration relies solely on vicarious techniques.

Although the use of the $654 \mathrm{~nm}$ channel was initially envisioned for 3MI, the option consisting of the former POLDER $765 \mathrm{~nm}$ broad channel remains under consideration depending on industrial feasibility and spectral stability that can be achieved for the proposed $754 \mathrm{~nm}$ channel. Therefore, in the present study we consider and compare both configurations. Similarly, the exact 3MI Instrument Spectral Response Functions (ISRFs) are not known yet, so we model the filters as perfect rectangular gates assuming thin and broad bandwidths as currently specified for $3 \mathrm{MI}$ in the mission requirement document. The in-band channel for MSPI is currently foreseen to be thinner than for 3MI with a spectral width of $6 \mathrm{~nm}$ and a central wavelength of $762 \mathrm{~nm}$. For both 3MI and MSPI the exact location of the out-band channel is not fully determined yet, so we simply used a spectral location that is completely free of $\mathrm{O}_{2}$ absorption.

Based on candidate channels illustrated in Fig. 1, three possible configurations (in-band/out-band for MSPI and inband/out-band or in-band/broad-band for 3MI) will be considered in the following analysis to evaluate which one provides the most information with respect to cloud top altitude (CTOP) and cloud geometrical thickness (CGT) parameters.

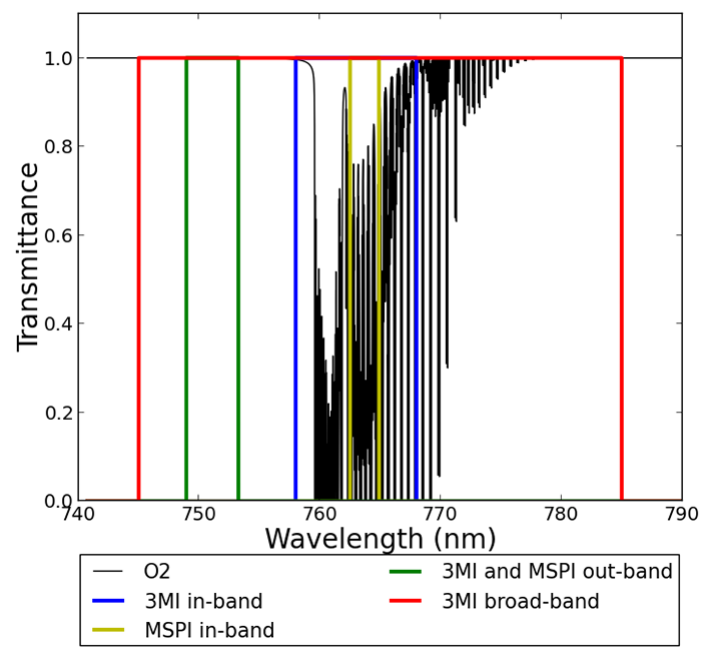

Figure 1. 3MI's filter models. The $\mathrm{O}_{2}$ transmittance is indicated by the black line. The green line represents the out-band filter (748.95$753.25 \mathrm{~nm})$, the red the broad-band $(745-785 \mathrm{~nm})$, the blue 3MI's in- or thin band (758-768 nm), and the yellow line MSPI's in-band (762.5-764.9 nm).

In this study, top-of-atmosphere (TOA) radiances are simulated using a radiative transfer code that accounts for gaseous absorption using a $k$-distribution technique (Kratz, 1995). Multiple-scattering effects are treated from the adding-doubling method (de Haan et al., 1987). In this approach, the radiative transfer equation is treated assuming a vertically inhomogeneous atmosphere (McClatchey model), stratified into plane and homogeneous layers and we only considered homogeneous single-layer clouds without aerosols. The $\mathrm{O}_{2}$ absorption is accounted for in and outside the cloud, including an interpolation of the $\mathrm{O}_{2}$ concentration when cloud base or cloud top altitude are between atmospheric levels.

For the sake of simplicity, our study is limited to the cases of homogeneous single-layer clouds without aerosols. Conclusions could be different in the presence of cloud inhomogeneity and three-dimensional effects, and the aerosols above the clouds, as they can have strong impacts on multi-angular measurements (Loeb and Coakley Jr, 1998; Várnai and Marshak, 2007; Di Girolamo et al., 2010; Liang and Girolamo, 2013). Furthermore, Heidinger and Stephens (2002) show that three-dimensional structures modify the photon mean path length; hence the $\mathrm{O}_{2}$ absorption in reflected A-band radiances, but it remains unclear how three-dimensional effects can modify its distribution when observed under multiangle geometries. In contrast, several theoretical, computational and observational studies have addressed the effects of three-dimensional cloud structure on transmitted A-band radiances and derived path length statistics; see Davis et al. (2009), Davis and Marshak (2010) and references therein. 


\section{$3 \mathrm{MI}$ view angles}
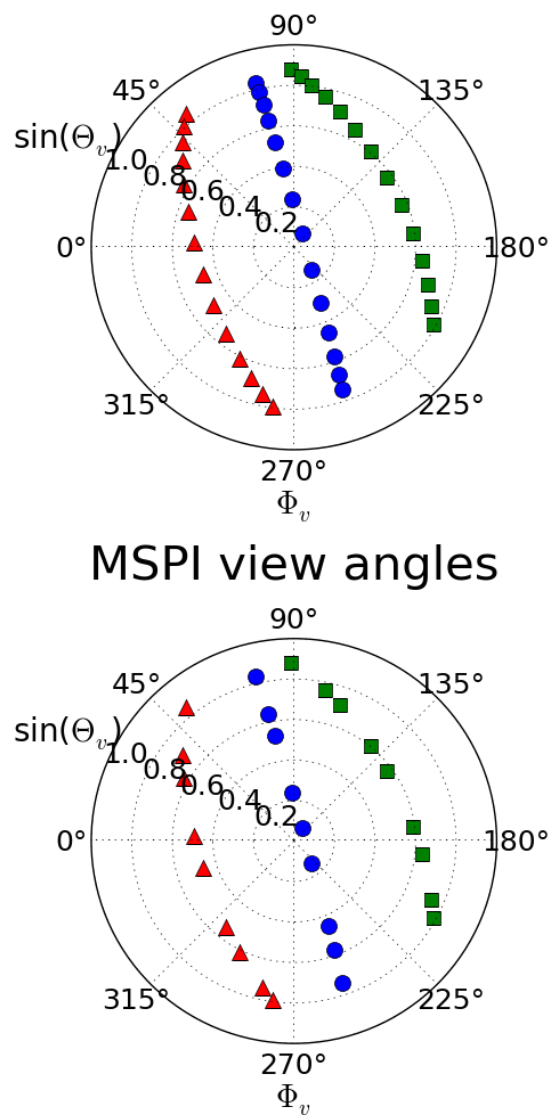

$\Delta \Delta$ AS $1 \bullet \bullet$ AS $2 \square \square$ AS 3

Figure 2. Angular sampling from the choice of MSPI's and 3MI's view angles.

\subsection{Angular configurations}

To limit the number of angular configurations, we chose to use three different realistic angular samplings (AS) described in Fig. 2 and precomputed for 3MI based on EP-SG anticipated orbit. We made this choice to obtain representative observational configurations. Indeed, with a solar zenith angle of $30^{\circ}$, for example, AS 3 corresponds to measurements in the backward scattering directions, AS 1 in the forwardscattering direction and AS 2 between them representative of along track sampling. The latter is also most representative of MSPI observation conditions due to its narrower swath compared to $3 \mathrm{MI}$.

\section{Sensitivity study of the A-band}

We first present, in this part, a qualitative sensitivity study of TOA simulated radiances to cloud geometrical thickness (CGT) and cloud top altitude (CTOP). As 3MI and MSPI configurations are not fundamentally different, here
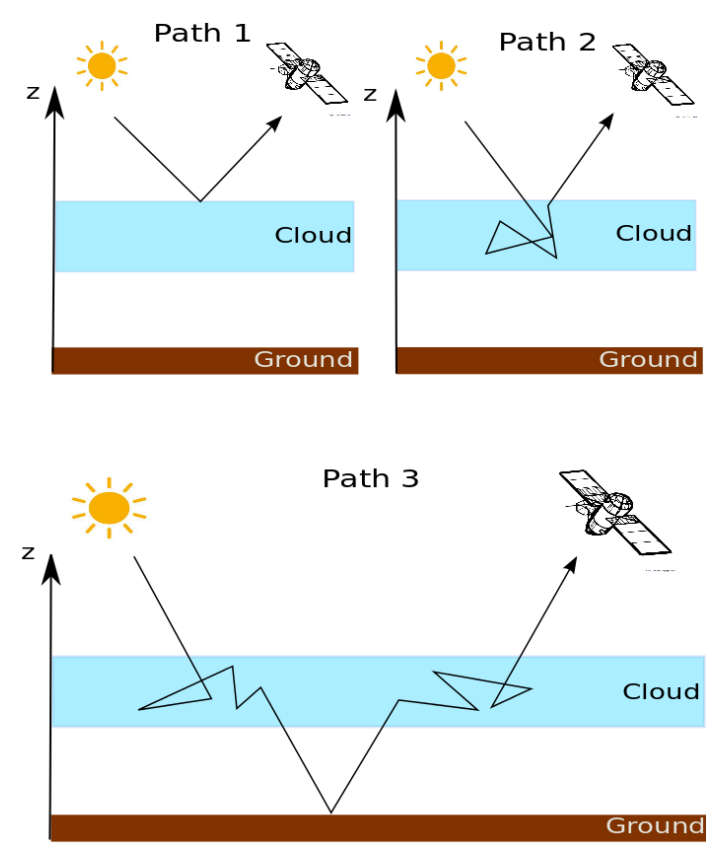

Figure 3. Basic pathways for a reflected photon through the atmosphere-surface system to reach the sensors. Molecular reflection which is weak is not represented.

we only use the $3 \mathrm{MI}$ 's thin-in/thin-out configuration to illustrate the main mechanisms, by which information on CTOP and CGT can be retrieved.

In addition to CGT and CTOP, the cloud optical thickness (COT) is also a key parameter. Indeed, previous studies shown that the A-band transmission is sensitive to it and provide limited information on CTOP for optically thin clouds (Desmons et al., 2013; Ferlay et al., 2010; Yang et al., 2013; Schuessler et al., 2014). Therefore, we will illustrate our discussion using three different cases of thin, moderately thin and thick clouds with optical thicknesses of 1, 4 and 16 respectively. The purpose of this first qualitative approach is to point out the main physical mechanisms that govern the $\mathrm{O}_{2}$ A-band TOA signals. Following this, a quantitative study will be developed and presented in the next section.

\subsection{Cloud top altitude and geometrical thickness sensitivity}

The A-band observation sensitivity to CTOP and CGT derives from the absorption by dioxygen occurring respectively above and inside the cloud. Knowing the vertical distribution of dioxygen in the atmosphere, the evaluation of absorption gives an indication of the photon geometrical path above and inside the cloud and, consequently, of CTOP and CGT.

Figure 3 shows, in a simplified way, the basic pathways that can follow a reflected photon in the atmosphere-surface system before reaching the sensors. A photon captured by the sensors can come from any of those paths. 
Path 1 represents photon reaching back from a scattering by the top layer of the cloud, path 2 from scattering inside the cloud and path 3 represents photons which have been reflected by the surface and have gone back through the cloud. When clouds are optically thick, as little radiation crosses the entire cloud, path 3 can be neglected. This explains why previous studies have usually been limited to thick clouds (Rozanov and Kokhanovsky, 2004; Davis et al., 2009; Ferlay et al., 2010; Yang et al., 2013).

In a first step, we considered the surface to be black, which is a good approximation of measurements performed over the ocean. In this case, contribution from path 3 is null. To illustrate the sensitivity of measurements to relevant parameters in a simple way, we represent the bands ratio for several top altitudes (CTOP) in Fig. 4 for three values of COT $(1,4,16)$ varying against the air mass factor. They are defined as follows:

$m_{\text {air }}=\frac{1}{\cos \theta_{\mathrm{v}}}+\frac{1}{\cos \theta_{s}}$

Figure 4 shows $\mathrm{O}_{2}$ A-band ratio in the solar plane with a solar zenith angle of $30^{\circ}$. The cloud geometrical thickness is fixed at $0.5 \mathrm{~km}$; the cloud droplet size distribution is chosen to be $\log$ normal with an effective radius of 12 microns and an effective variance of 0.02 . The different coloured curves represents different values of CTOP (from 1 to $6 \mathrm{~km}$ ). For each air mass we obtain two values of the ratio: the upper part corresponds to the backward scattering direction and the lower part to the forward-scattering direction.

The two branches of the curves correspond to the forwardand backward-scattering halves of the principal plane. The latter shows a distinctive angular feature in the nearbackward-scattering direction that is traceable to the phase function, which diminishes as COT increases. Evidently, the A-band ratio decreases generally as the air mass factor; hence the absorption increases. A variation of CTOP leads to a variation of path 1 directly (Fig. 3). By increasing CTOP, the photon path length above the cloud is decreased. The absorption of the in-band is thus reduced whereas the one of the out-band is not affected by absorption. Consequently, the ratio increases with CTOP, as can be seen in Fig. 4. We note that ratio change due to CTOP variation is almost the same for any angle so no angular signature of CTOP changes. Indeed, for optically thick clouds, the band ratio decreases linearly with the air mass, which is the expected behavior when the cloud is assumed to be a solid reflector.

Figure 5 is the same as Fig. 4 but with a fixed cloud top altitude $(6 \mathrm{~km})$ and for varying geometrical thickness CGT (from 1 to $5 \mathrm{~km}$ ). It corresponds to a variation of path 2 as the CTOP is constant and the surface black. The ratio decreases as CGT increases. Indeed, for a fixed CTOP and constant COT, an increase of CGT leads to a decrease of the extinction within the cloud. It yields a deeper penetration and an enhanced mean free path of the photon and therefore an in-
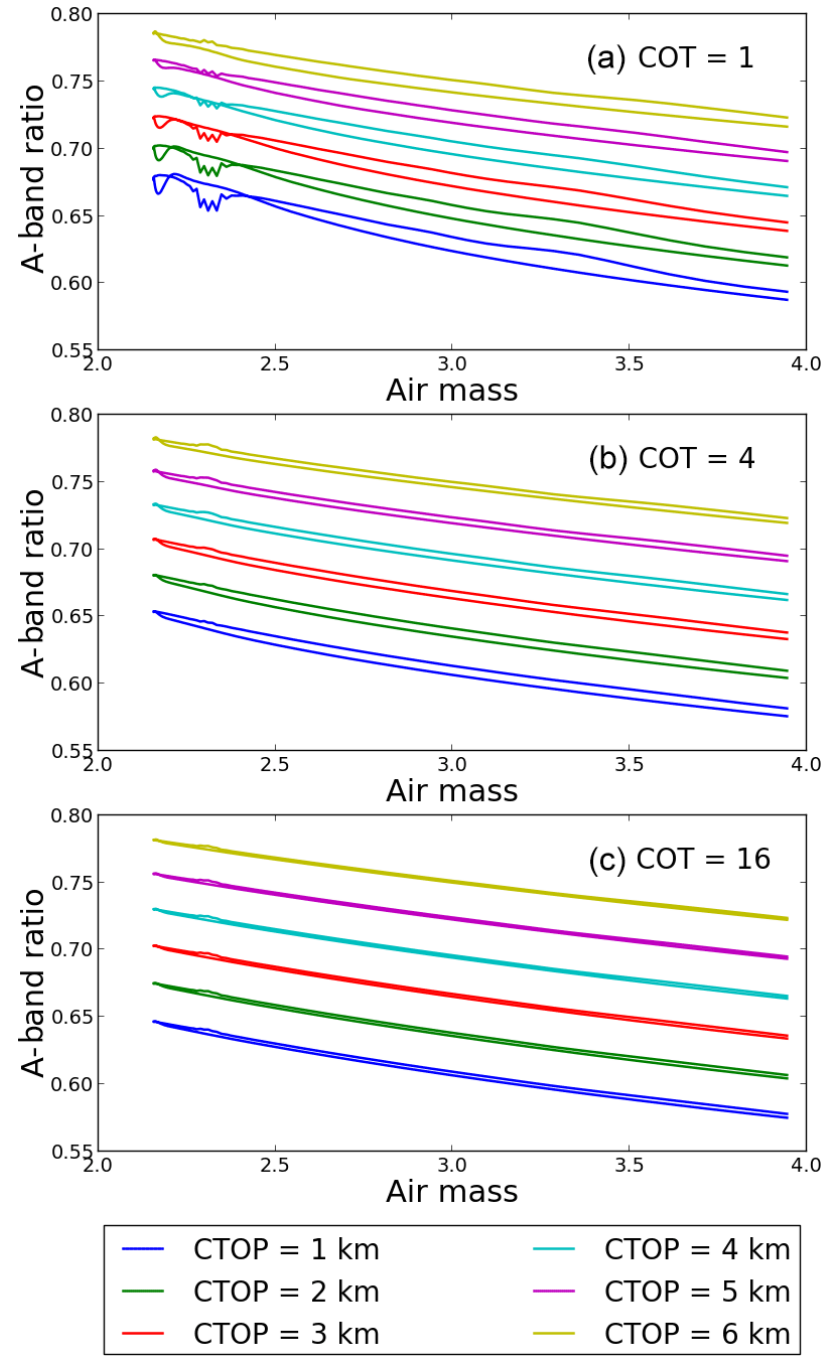

Figure 4. Effect of cloud top altitude variation CTOP on the band ratio. $\mathrm{CGT}=0.5 \mathrm{~km}, \mathrm{SZA}=30^{\circ}$, $\mathrm{VAA}=0^{\circ},(\mathbf{a}) \mathrm{COT}=1,(\mathbf{b}) \mathrm{COT}=4,(\mathbf{c}) \mathrm{COT}=16$.

crease of absorption in the affected channel and a decrease of the ratio.

For COT $=1$, we notice differences between forward and backward scattering which are linked to cloud particle microphysics through their phase function asymmetry factor. The photon path length is, in case of low COT, longer in the forward view direction, so larger in the absorption. This effect is more pronounced when CGT increases because, for a fixed COT, the extinction within the cloud decreases leading to a deeper penetration inside the cloud. Variations of the ratio due to CGT changes are thus significantly angularly dependent for the case of an optically thin cloud. Thus, as can be seen on Figs. 4 and 5, the ratio of reflectances, which provides oxygen transmittance, depends on CTOP and CGT variation. This double sensitivity raises the question of the retrieval of CTOP without knowledge of CGT and of the si- 

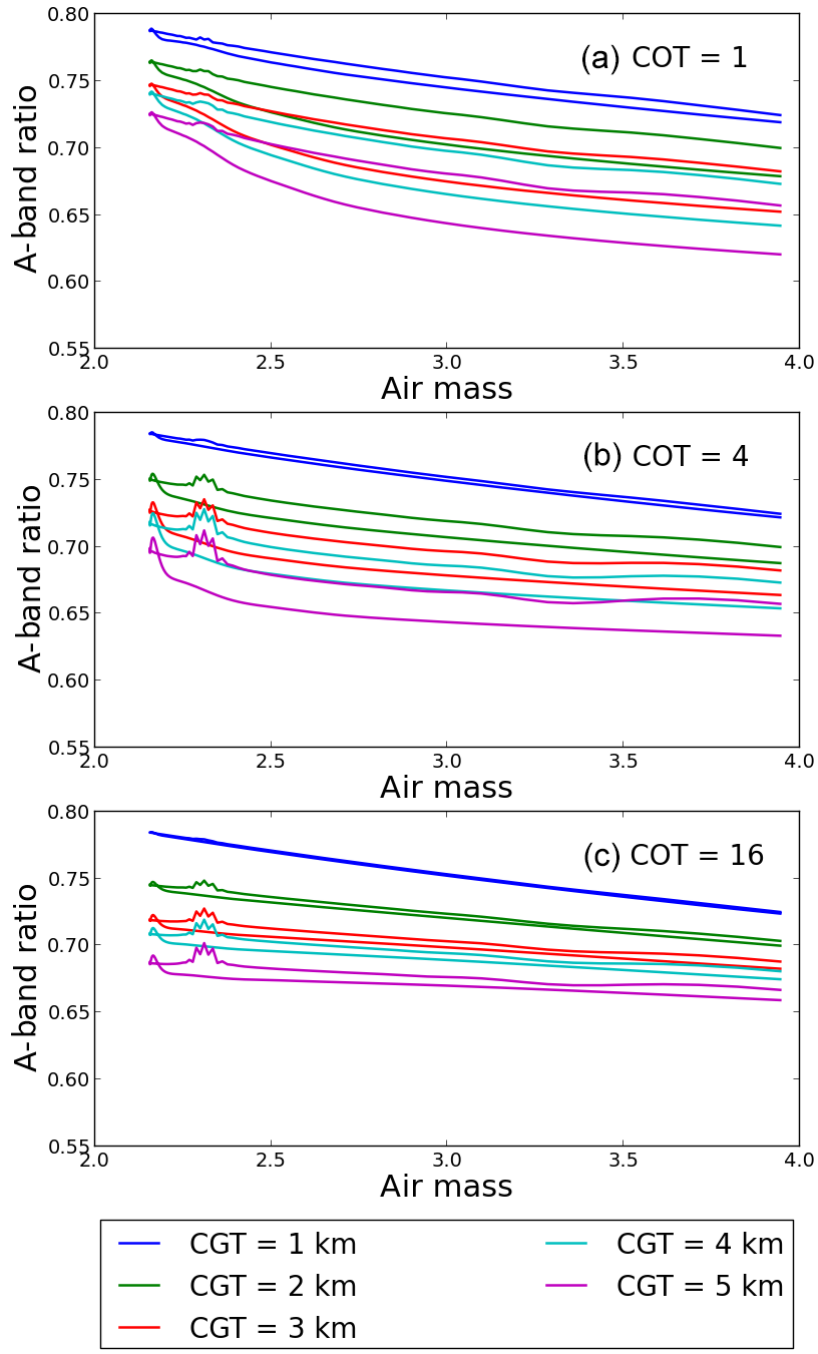

Figure 5. Effect of cloud geometrical thickness variation CGT on the band ratio. $\mathrm{SZA}=30^{\circ}, \mathrm{VAA}=0^{\circ}, \mathrm{CTOP}=6 \mathrm{~km},(\mathrm{a}) \mathrm{COT}=1$, (b) $\mathrm{COT}=4$, (c) $\mathrm{COT}=16$.

multaneous retrieval of both CTOP and CGT. One way to address these issues could be in the information gained by the angular dependance of the reflectance ratio on CGT and CTOP, as already noted by Ferlay et al. (2010) for optically thick clouds. The average value of $\mathrm{O}_{2}$ A-band ratio is directly linked to the mean photon path length which to a first order is determined by the cloud top altitude, hence the shift of the curves in Figs. 4 and 5 for the different CTOP values. In order to summarize the angular measurement variations, following Ferlay et al. (2010), who shows that the standard deviation of the oxygen pressure is linearly linked with the geometrical thickness, we use the standard deviation of the $\mathrm{O}_{2}$ A-band ratio. Note that this metric does not retain all the information contained in the angular variation of the signal, but provides a useful qualitative link to previous studies by Ferlay et al. (2010). Physically, as will be discussed later on, the angular variation of the signal is linked to varying photon penetration within the cloud which depends on cloud extinction (or CGT as we consider COT as fixed and the cloud as homogeneous), air mass factor and details of the cloud particle phase function (through primarily their asymmetry factor). To first order, the larger photon penetration will result in a larger dependence on air mass factor, hence a larger standard deviation of angular measurements.

For optically thick clouds (COT $=16$, Fig. $5 \mathrm{c}$ ), the difference between forward and backward scattering is lowered and is practically null for small geometrical depth as this corresponds to the solid reflector situation already described in Fig. 4c.

For the other case with COT $=4$ (Fig. 5b), we notice optically thin cloud characteristics for large CGT (differences between forward and backward scattering) and thick clouds characteristics for low CGT. As will be discussed later, this actually reflects the transition from single-to-multiplescattering regimes as the extinction within the cloud increases.

To illustrate the angular A-band ratio dependance on CTOP and CGT, Fig. 6 presents isolines of CTOP and CGT values in a two-dimensional space with average band ratio value $R$, here along the ordinate axis, and the angular standard deviation $\left(\sigma_{R}\right)$ along the abscissa axis. Values are obtained for the AS 1 (cf. Fig. 2) for 3MI's 14 view angles using same cloud parameters as for Figs. 4 and 5. In practice, we represent here a solution space from which one could attempt to retrieve CTOP and CGT knowing $R$ and $\sigma_{R}$. Figure $6 \mathrm{a}, \mathrm{b}, \mathrm{c}$ show the solution space for COT of 1,4 and 16 respectively.

In Fig. $6 \mathrm{a}(\mathrm{COT}=1)$, we notice that the curves of constant CTOP (red curves) and CGT (black curves) are well separated. The curves of constant CGT are almost vertical, which means that CTOP is primarily sensitive to $R$ and less dependent on $\sigma_{R}$. Indeed, as explained before, CTOP variations imply a change of molecular absorption above the cloud, which acts principally on the A-band ratio average (Fig. 4). Conversely, constant CTOP curves are almost horizontal traducing the fact that CGT is mostly sensitive to $\sigma_{R}$ only. The modification of CGT for constant optical thickness produces a change of extinction inside the cloud and of the penetration depth, which causes a stronger angular dependence as the photon path length in the cloud depends on the view direction (Fig. 5). Even though the solution space is not orthogonal, this figure shows how $R$ and $\sigma_{R}$ could be used to infer both CTOP and CGT. For optically thick clouds (Fig. 6c), the ratio is again sensitive to both parameters but it is important to note that, contrary to small COT, increasing CGT here leads to smaller $\sigma_{R}$. We can relate this observation to Fig. 5c, in which the slope of A-band ratio decreases with CGT, thereby reducing its angular standard deviation. On the contrary, for optically thin clouds and in Fig. 5a, the slope increases when CGT increases (at least for low air mass). This inverse sensitivity of $\sigma_{R}$ implies that, between the cases of optically thin and thick clouds, CGT no longer becomes 

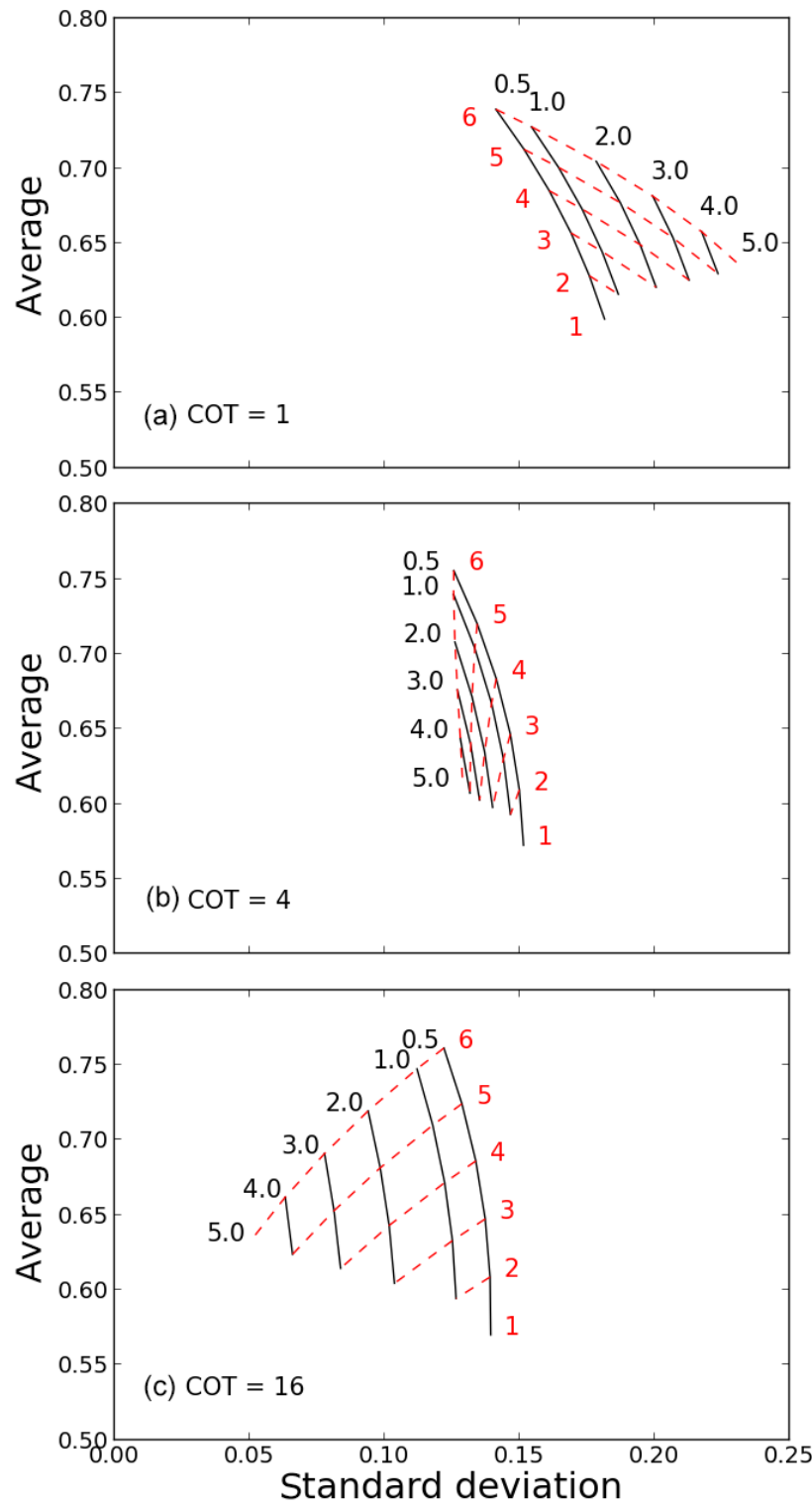

$\ldots \mathrm{CTOP}(\mathrm{km})-\mathrm{CGT}(\mathrm{km})$

Figure 6. Look-up tables for different cloud top height CTOP and cloud optical thickness CGT, simultaneously of average and angular standard deviation of the 3MI's A-band ratio. In red, CTOP values and in black, CGT values: (a) COT $=1$, (b) $\mathrm{COT}=4$, (c) $\mathrm{COT}=16$.

sensitive to the standard deviation. At this intermediate optical depth, almost no radiation crosses the cloud completely, as it would for COT $=1$, but the signal is neither saturated by multiple scattering as is the case for COT $=4$. Consequently, the A-band ratio varies with both COT and CGT in almost the same way at every angle leading to no variation of the standard deviation. That is confirmed by Fig. $6 \mathrm{~b}$ $(\mathrm{COT}=4)$ where both parameters are sensitive primarily to
$R$ and the solution space collapses along the $\sigma_{R}$ axis. This correlation between the sensitivities make it impossible to infer both parameters from the reduced set of $R$ and $\sigma_{R}$ metrics. This second qualitative analysis again illustrates how multi-angle A-band ratio measurements above a black surface can potentially convey information on both CTOP and CGT for optically thick clouds, as was already noticed by Ferlay et al. (2010), but also for thin clouds. Between these two cases, however, the reduced set of metrics based on Aband differential absorption measurement does not seem to allow simultaneous retrieval of CTOP and CGT.

\subsection{Addition of a Lambertian surface}

A black surface is a good approximation of the ocean out of the sun glint area. However, for retrievals over land surfaces, we need to extend our model and analysis to include more realistic surface reflectance. To focus on the physical understanding of mechanisms contributing to the band ratio signal, we choose to simulate the surface by using a simple Lambertian model with two very different albedo values: 0.2 (corresponding approximatively to sand) and 0.8 (fresh snow or ice), thus activating path 3 in Fig. 3 .

Figure 7 is the same as Fig. 4 with a surface albedo of 0.2 . For $\mathrm{COT}=16$, the cloud opacity is such that the impact of the surface is negligible. For COT $=1$ and 4, differences are notable. The ratios are weaker. Because of surface brightness, the probability of longer path length increases, further attenuating radiation in absorbing channels. Indeed, the non-absorbing spectral bands present higher reflectance thanks to the reflection, which can be multiple, between the surface and the cloud. Such reflection for the absorbing band also occurs but the increased absorption limits their contribution to observed TOA reflectance. By adding a bright surface, the out-band radiance increases more than the in-band one, thereby the A-band ratio is reduced. This effect is more important for high cloud top altitudes and low air mass. Indeed, the higher the cloud, the more the photon pathway between the surface and the cloud increases. The difference between in- and out-band, therefore, increases and the band ratio decreases. For COT $=1$ and low air mass, it logically appears that the sensitivity to CTOP is extremely low as the cloud does not significantly modify the photon pathway.

Figure 8 is the same as Fig. 5 with a surface albedo of 0.2. Again, a cloud with COT $=16$ (Fig. $8 \mathrm{c}$ ) is very opaque and the surface has almost no effect in the oxygen A-band ratio. For $\mathrm{COT}=4$ and $\mathrm{COT}=1$, the ratio is also lower. The decrease is higher here for geometrically thick clouds due to the same effect seen through. The multiple reflections between the cloud and the surface increases the photon path, hence the absorption in the in-band. The important parameter to consider is thus the cloud base altitude. The higher the cloud base, the more the surface affects the A-band radiance because of the difference between in- and out-band radiances. 

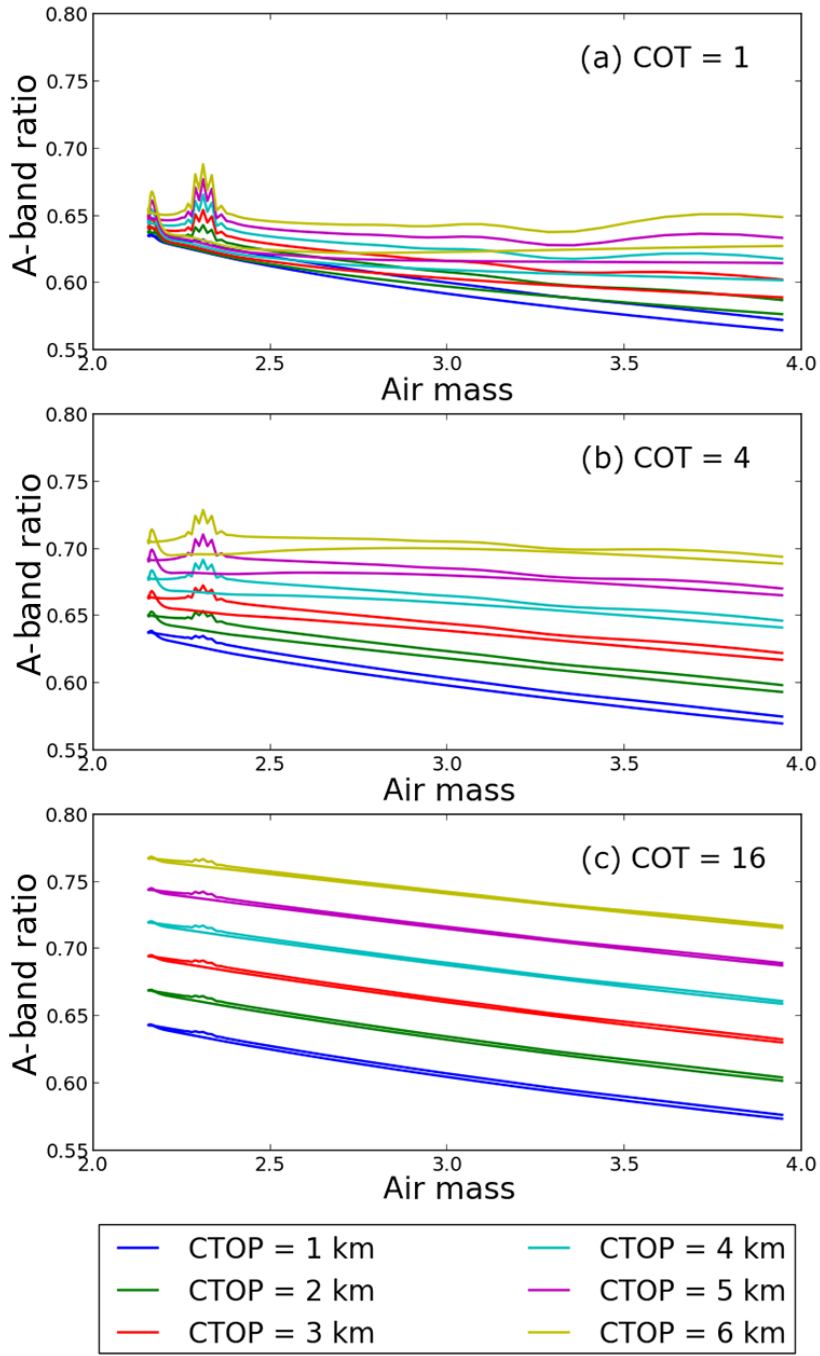

Figure 7. Same as Fig. 4 with a surface albedo of 0.2. (a) $\mathrm{COT}=1$, (b) $\mathrm{COT}=4$, (c) $\mathrm{COT}=16$.

Figure 9 is the same as Figs. 4 and 7 and Fig. 10 as Figs. 5 and 8 with a surface albedo of 0.8 . With a brighter surface, the effect of the surface is obviously more apparent. For $\mathrm{COT}=1$, the cloud altitude and thickness have almost no impact on the A-band ratio because the radiance coming from the cloud represents a negligible part of the signal. For $\mathrm{COT}=16$, the cloud signal is higher; consequently, the ratio is modified depending on CTOP and CGT.

To summarize, a Lambertian surface decreases A-band sensitivities on both CTOP and CGT for the smallest COT, the COT limit depending on the surface albedo.

\section{Theoretical information content study}

In order to go further with the sensitivity studies on the $\mathrm{O}_{2}$ A-band, we performed an information content study based on a Bayesian optimal estimation approach. It also provides
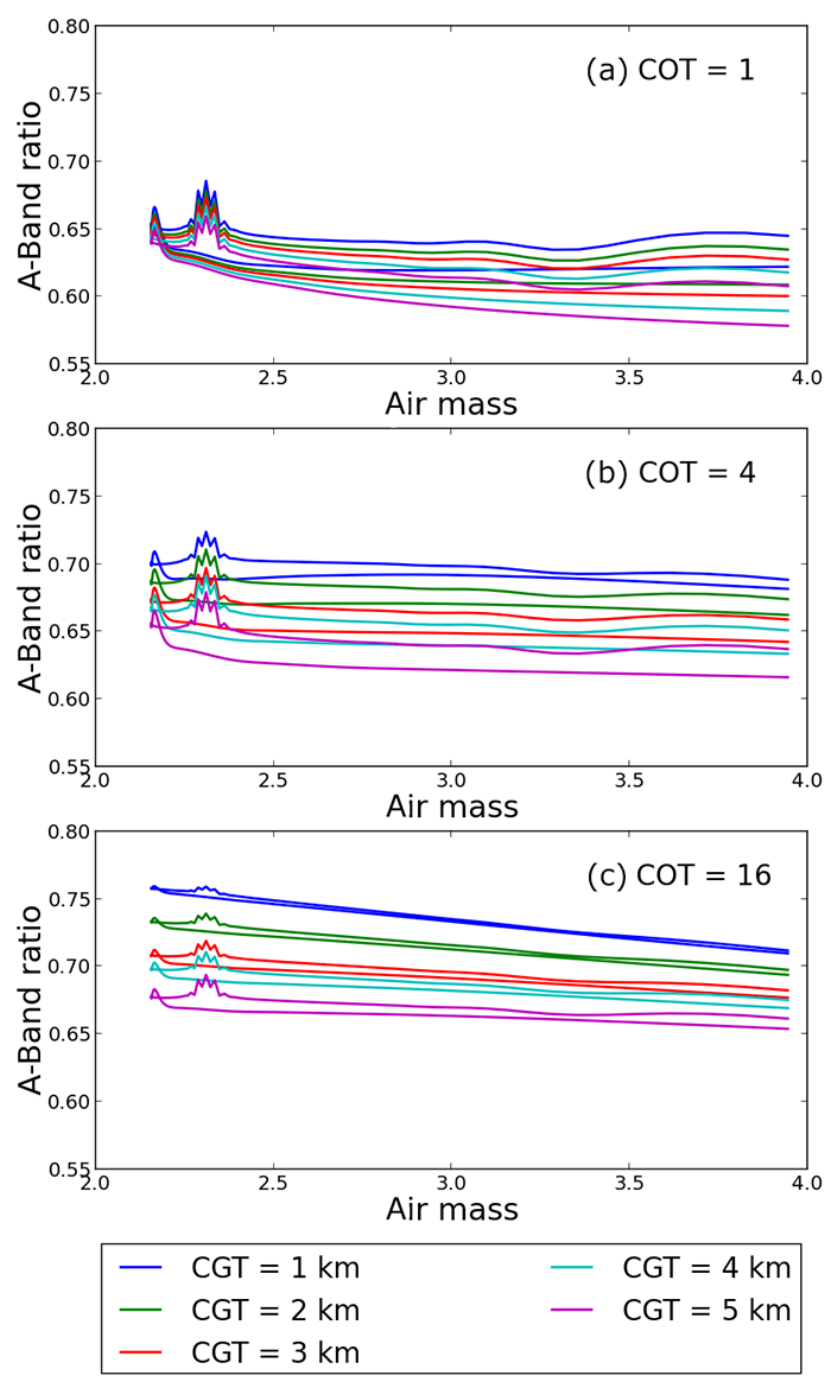

Figure 8. Same as Fig. 5 with a surface albedo of 0.2. (a) $\mathrm{COT}=1$, (b) $\mathrm{COT}=4$, (c) $\mathrm{COT}=16$.

an elegant framework to compute the information content of the observing system as well as to perform an error analysis on the resulting retrieval. The formulation of the problem in this study has been developed by Rodgers (1998) and used in previous studies by several authors (Sourdeval et al., 2013, 2014; King and Vaughan, 2012; Cooper et al., 2006).

\subsection{Information content theory}

Details on how the information content is calculated can be found in the aforementioned studies and, for the sake of completeness, the main theoretical elements are reported in Appendix A. In this section, we simply describe and specify the different parameters used in the Rodgers information content formulation.

For this study, the state vector $\boldsymbol{x}$ contains the parameters CTOP and CGT and the measurement vector, A-band ratios 

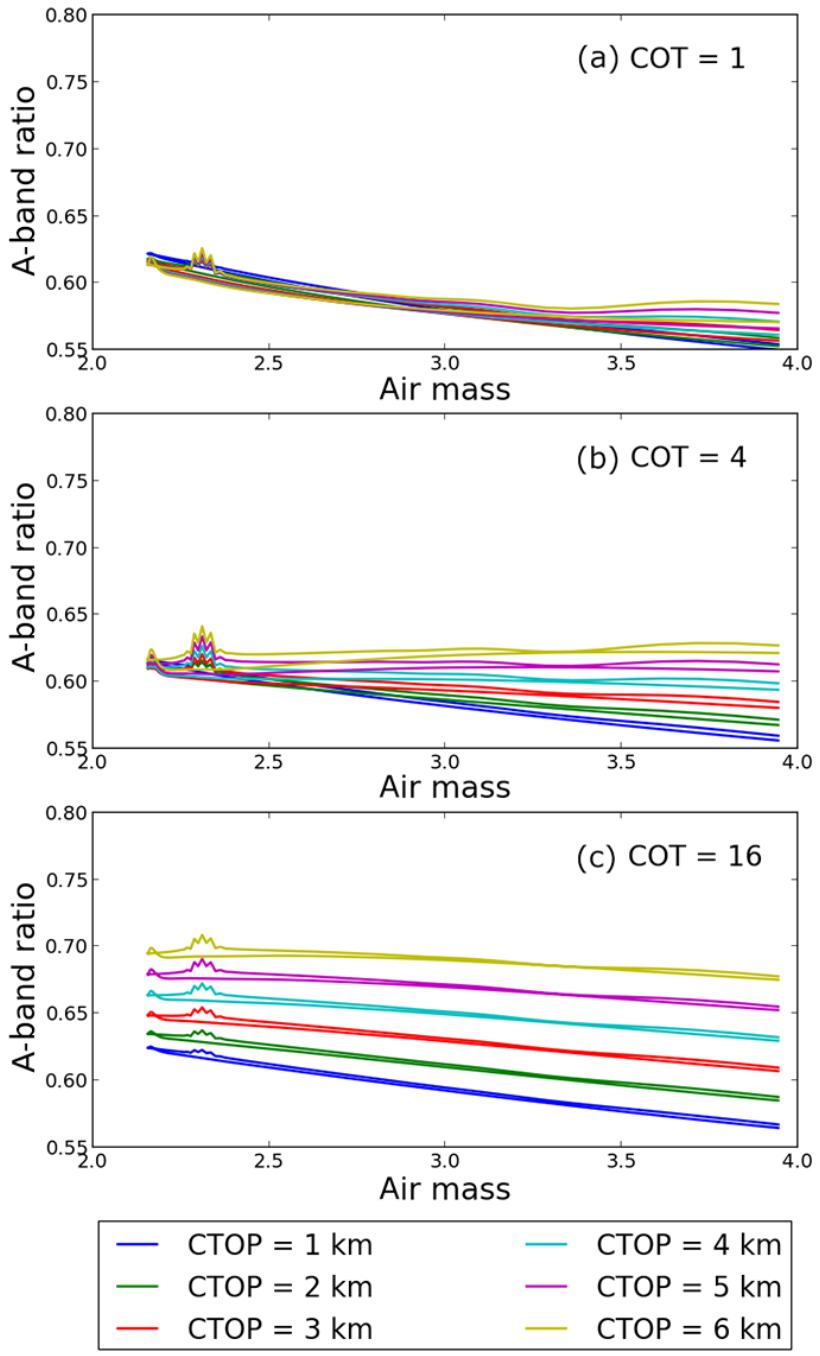

Figure 9. Same as Figs. 4 and 7 with a surface albedo of 0.8. (a) $\mathrm{COT}=1$, (b) $\mathrm{COT}=4$, (c) $\mathrm{COT}=16$.

for the different view angles described in Fig. 2. As for nonretrieved parameters, we considered only COT, the effective radius of the cloud particles and the surface albedo. Preusker and Lindstrot (2009) showed that those parameters are indeed the geophysical quantities that most influence the measured A-band ratio.

The only information we have a priori is that cloud top altitude CTOP cannot be very high and cloud thickness CGT cannot exceed CTOP. Consequently, we considered an a priori standard deviation of $5 \mathrm{~km}$ for both CGT and CTOP for both liquid and ice clouds. The a priori variance-covariance matrix $\mathbf{S}_{a}$ is assumed diagonal and elements are those variances $\left(\mathbf{S}_{a, i i}=5^{2}, i=1,2\right)$. This represents our knowledge of the state vector before the measurements and retrievals are performed. Those a priori (co)variance values do not have a strong impact on the information content as they are chosen to be very large compared to the a posteriori variances $\left(\mathbf{S}_{x}\right)$.
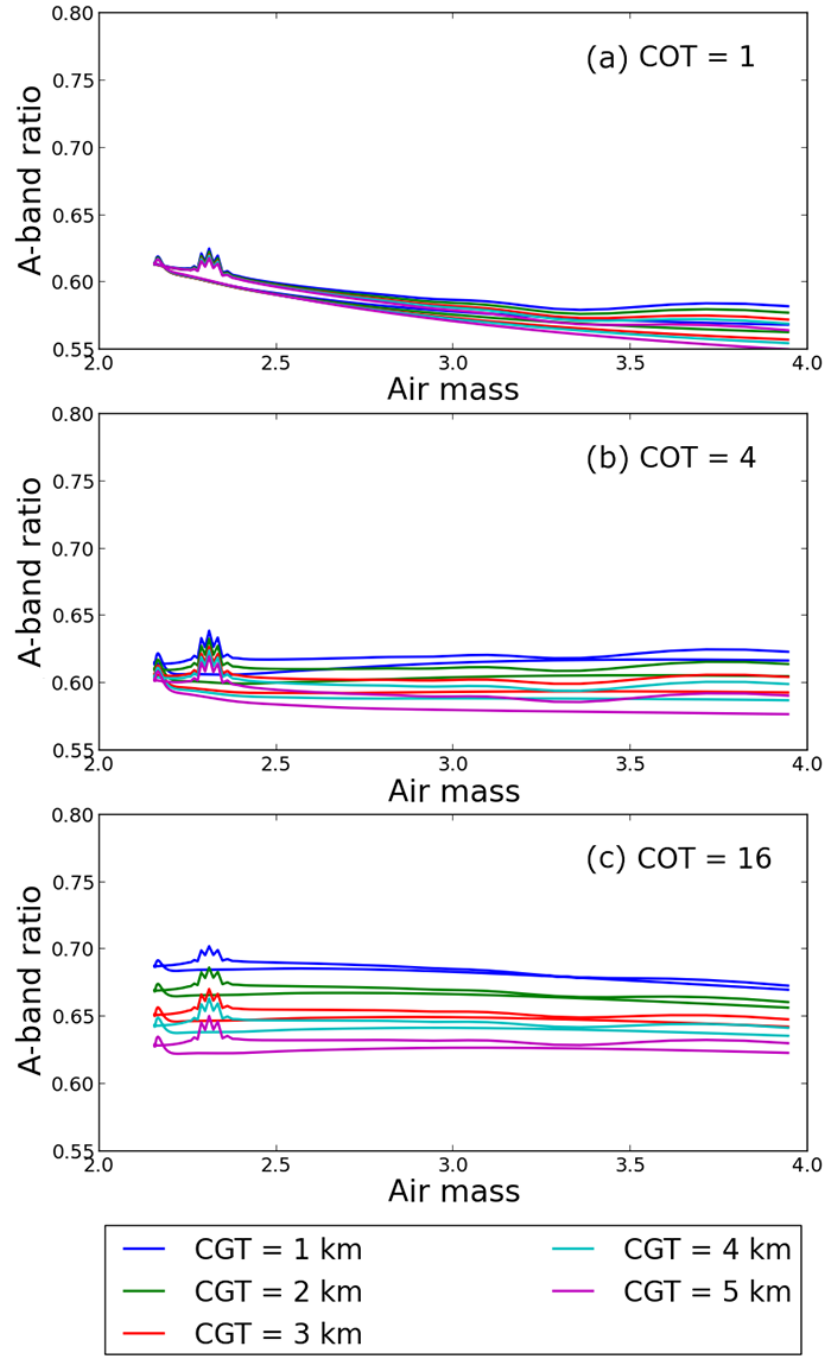

Figure 10. Same as Figs. 5 and 8 with a surface albedo of 0.8. (a) $\mathrm{COT}=1$, (b) $\mathrm{COT}=4$, (c) $\mathrm{COT}=16$.

Measurement noise is assumed to be $1 \%$ of the ratio for both MSPI and 3MI. Indeed, the ratio method leads to a smaller noise, which is typically considered to be of the order of $2-3 \%$ for the radiances themselves. The uncertainty used for the band ratio is smaller than the uncertainty for individual channels because the calibration of the two channels can be performed directly by relating their ratio to the apparent surface pressure, which is considered to provide higher accuracy than what can be achieved for absolute calibration of single-channel measurements (Fougnie, 2016). We use this measurement noise estimate to compute a diagonal variancecovariance matrix as

$\mathbf{S}_{y, i i}=\left(0.01 \times \frac{\operatorname{Rad}_{\text {in }, i}}{\operatorname{Rad}_{\text {out }, i}}\right)^{2}$ 


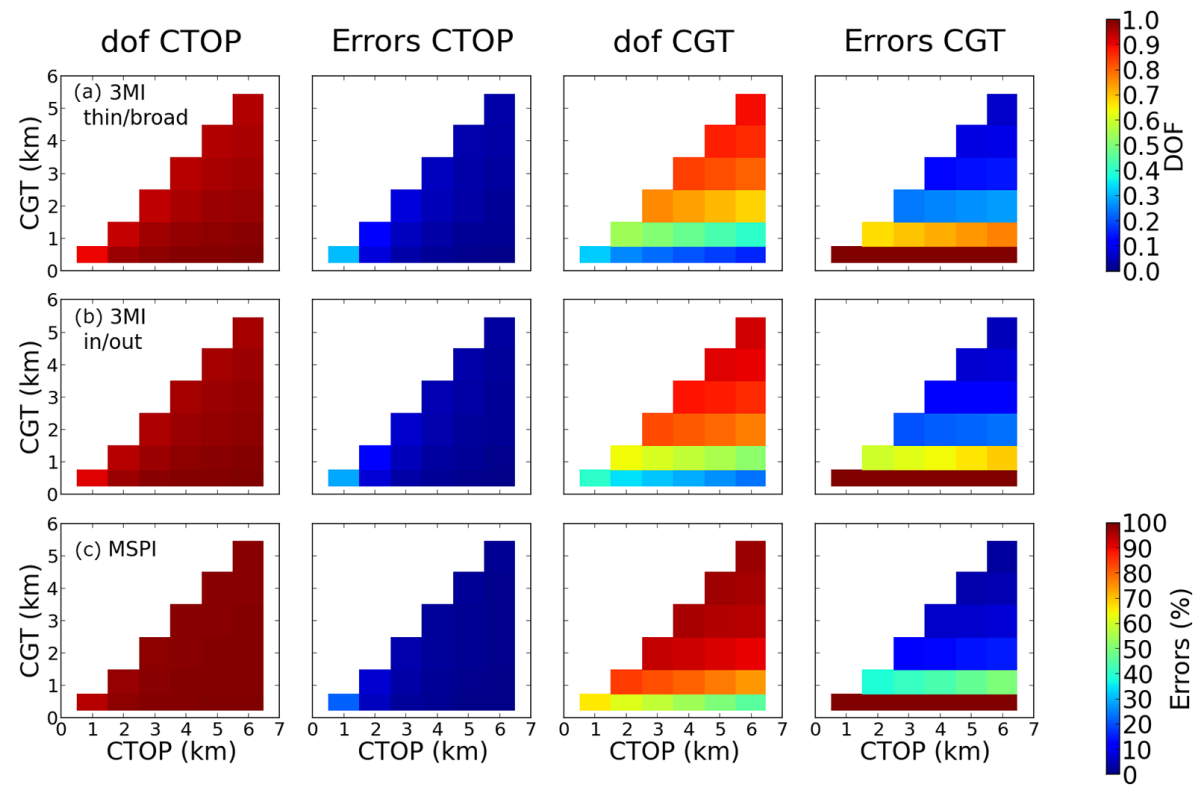

Figure 11. 3MI/MSPI information content comparison. Partial DOFs and a posteriori errors of the A-band of (a) 3MI with the configuration using thin band on broadband, (b) 3MI with the in-band on out-band method and (c) MSPI, using the in-band on out-band method with a different filter and 9 view angles instead of 14 for $3 \mathrm{MI}$. COT $=16, r_{\text {eff }}=12 \mu \mathrm{m}, v_{\text {eff }}=0.02, \mathrm{SZA}=30^{\circ}$, orbit $/ \mathrm{AS} 1$.

for the $i$ th angular measurement, where subscript "out" means either the continuum channel (MSPI or 3MI) or 3MI's broadband channel.

We introduce also a variance-covariance matrix for the forward model, linked to the non-retrieved parameters. Those parameters are fixed and assumed known but only to within a known uncertainty. We considered $10 \%$ relative errors on COT and the effective particle radius and an absolute error of 0.05 on the surface albedo.

\subsection{Intercomparison of 3MI and MSPI - measurement information content}

We use the formalism in Appendix A to study the information content of the three different $\mathrm{O}_{2}$ A-band measurement configurations described in Sect. 2.3 and perform an intercomparison for a reference case. The reference case is a homogeneous liquid cloud with an optical thickness of 16, with droplets following a log-normal distribution with an effective radius of $12 \mu \mathrm{m}$ and an effective variance of 0.02 . The surface is assumed black, SZA is fixed at $30^{\circ}$, and we use the angular sampling configuration AS 1 (cf. Fig. 2). We describe and compare the A-band information content provided by the three instrumental configurations by analysing the partial degrees of freedom and the a posteriori errors (square roots of the diagonal elements of the a posteriori variance-covariance matrix $\mathbf{S}_{y}$, as described in Appendix A).

Figure 11 presents an example of the information content of the A-band observations obtained from the three instrumental configurations. In this figure, we have plotted the de- grees of freedom (DOFs) and the a posteriori errors in the state space: CGT on the vertical axis for six values ranging from 0.5 to $5 \mathrm{~km}$ and CTOP on the horizontal axis for six values ranging from 1 to $6 \mathrm{~km}$. We note that, logically, the higher the information content, the lower the a posteriori errors. We also notice some general similarities between the three configurations. The A-band information on CTOP increases when it increases and CGT decreases. It is the reverse for the information content on CGT that increases with a decrease of CTOP and an increase of CGT. Indeed, when the information on the more sensitive parameter (CTOP) decreases, a part of the lost information is reported to the other parameter (CGT). For the cases with small CGT, there is low information content, showing us that the retrieval of this parameter is not reliable (i.e. $\mathrm{DOF}<0.5$ ).

Comparison of Fig. 11a, b and $\mathrm{c}$ (the three rows) shows that information content of 3MI (thin band/broadband) is lower than 3MI (in-band/out-band) that is in turn lower than MSPI. The in/out-band configuration is thus more effective than the thin band/broadband one. Even if MSPI has fewer view angles than 3MI, it provides higher information. We can therefore conclude that MSPI's spectral channel definitions are more suitable than those of 3MI for the current application.

The DOF (per retrieved parameter) and a posteriori error are obviously linked. Figure 12 represents the relationship between the degrees of freedom and the a posteriori errors (in percent on CTOP and on CGT). As expected, the a posteriori errors decrease with the degree of freedom since the intake of information decreases the errors on the retrieved parameters. 

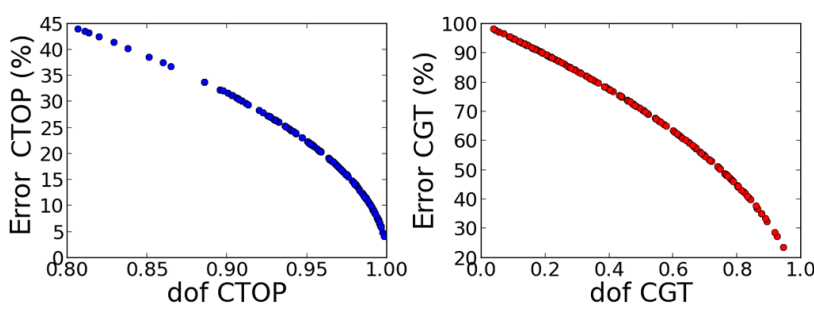

Figure 12. Relation between the DOFs and the a posteriori errors on CTOP (left) and on CGT (right).

In the following, we will therefore only consider the DOFs. Moreover, respective performances and differences between those spectral configurations are fairly invariant for the cases studied. For analysis of multi-angle observation, we therefore only present and discuss results for $3 \mathrm{MI}$ thin/broad configuration. This provides a baseline evaluation of the (minimum) available information from multi-angle $\mathrm{O}_{2}$ A-band measurements, knowing this could be improved through adjustment of the spectral channel definition (position and bandwidth).

At this stage, it should be noted though that the impact of spectral configuration in terms of information content remains smaller than the additional information provided by multi-angular measurements when compared to single-view observations. To illustrate this, we have studied the information available from mono-angular vs. multi-angular Aband ratio measurements which are illustrated by Fig. 13 . Unlike other examples, we choose here to use MSPI's Aband ratio for this comparison to illustrate what can actually be achieved from single-view measurements in a favourable case where spectral configuration is optimized. Here the mono-view scenario is nadir-looking $\left(0^{\circ}\right.$ zenith angle) and the multi-view assumption is the same as the case illustrated by Fig. 11. Figure 13a shows the information content on CTOP and CGT from mono-angular measurement. In this case, the information on CTOP is high but the A-band ratio does not bring information on CGT. As expected, retrieving two parameters with only one measurement is simply not possible, and this is clearly reflected by the result. But the retrieval of CTOP appears indeed possible without any knowledge of CGT, as already noticed by Preusker and Lindstrot (2009). Even so, the information on CTOP is lower than MSPI's multi-angular measurements (Fig. 11).

In a second step, we evaluated whether information could be obtained for CGT if a priori knowledge is used for CTOP. For this purpose, we decreased the a priori variance on CTOP to $0.5 \mathrm{~km}$, corresponding approximately to the accuracy of a retrieval using the $\mathrm{CO}_{2}$-slicing method (Platnick et al., 2003). By doing this, we determine the information content brought by the A-band ratio on CGT, knowing CTOP with such accuracy. Figure 13b represents this information content for mono-angular measurement and Fig. 13c for MSPI's multiangular measurements. The results clearly illustrate that information content of mono-angular A-band ratio on CGT is

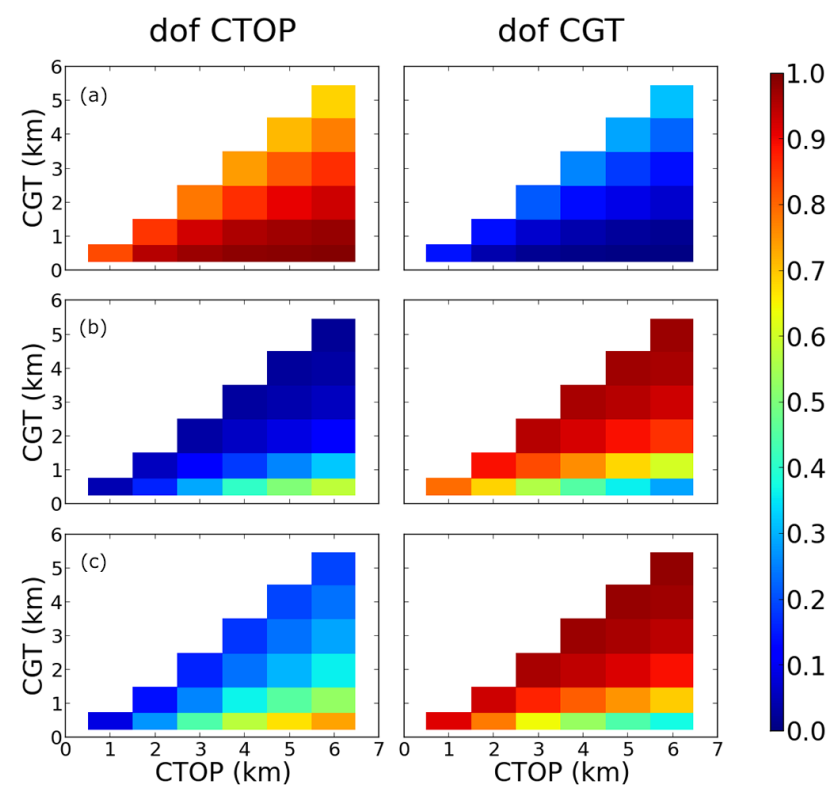

Figure 13. Comparison between mono- and multi-angular A-band ratio with MSPI's configuration: (a) mono-angular A-band ratio $\mathrm{DOF}$ (VZA $=0^{\circ}$, nadir), (b) same as (a) with a reduced a priori standard deviation on CTOP $(0.5 \mathrm{~km})$ and (c) same as (b) for multi-angular measurements (orbit/AS 1). COT $=16, r_{\text {eff }}=12 \mu \mathrm{m}$, $v_{\text {eff }}=0.02, \mathrm{SZA}=30^{\circ}$.

comparable (though still smaller) to what is available from MSPI, pending the fact that sufficient a priori knowledge is available on CTOP. We also notice that the A-band ratio brings some limited additional information to CTOP for low CGT and high CTOP. It means that A-band ratio is able to reduce the a priori volume even if this one is already small. Again, and as already discussed by Preusker and Lindstrot (2009), these results simply illustrate that mono-angular Aband measurements can be used to retrieve CTOP without knowledge of CGT. When a priori knowledge on CTOP is provided, CGT could potentially be retrieved from singleview observation.

This conclusion should however be considered in view of our present assumption that other non-retrieved parameters, such as COT or particle size, are quite well known. We then anticipate that multi-angle measurements will be more robust to noise but it remains to be investigated how the various errors on measurements and non-retrieved parameters impact the a posteriori errors on CGT for single-view or multiangular observations. This issue is clearly outside the scope of our present study since we focus on multi-angle measurements to simultaneously retrieve CTOP and CGT with no further a priori information on either (beyond that encapsulated in $\mathbf{S}_{a}$ ). 


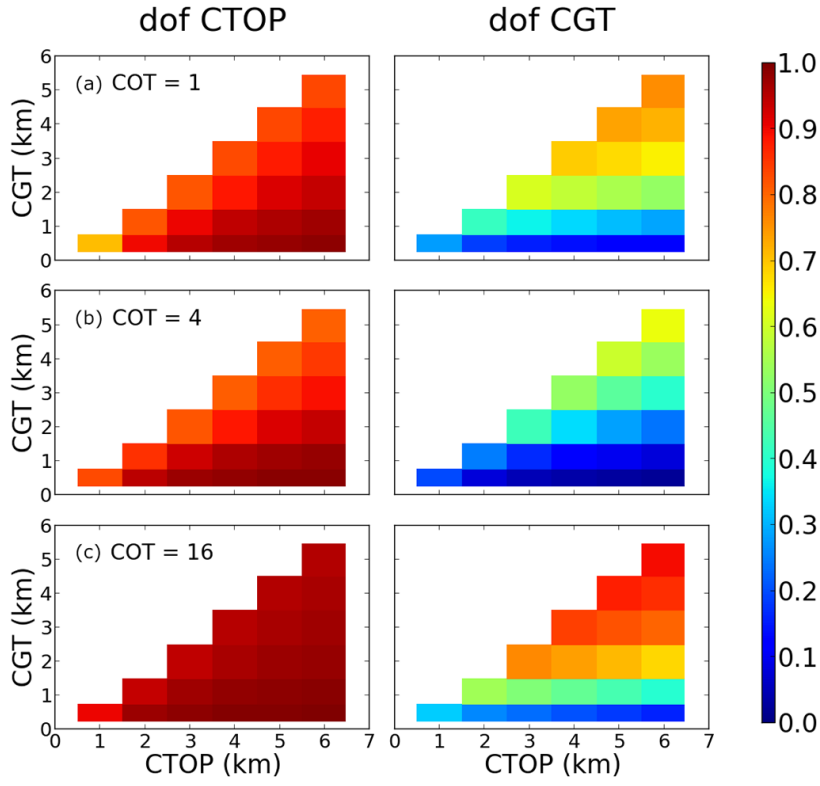

Figure 14. Information content of 3MI A-band for different COTs: (a) $\mathrm{COT}=1$, (b) $\mathrm{COT}=4$, (c) $\mathrm{COT}=16$. Partial DOFs for CTOP (left) and CGT (right).

\subsection{Cloud optical thickness variation}

The effect of the optical thickness on measurement sensibilities were examined previously. Here, we study it in terms of information content in order to take into account the measurement noise and uncertainty in non-retrieved parameters (COT and effective radius, the surface being considered black in this case).

Figure 14 shows information content of 3MI A-band ratio for the reference case as well as for several other optical thicknesses (same ones as in Sect. 3). The information content is, for optically thick $(\mathrm{COT}=16)$ or thin $(\mathrm{COT}=1)$ clouds, pretty close and significantly higher than for the intermediate optical thickness $(\mathrm{COT}=4)$. This confirms the finding in Fig. 6, where the LUT representation shows that retrieval of both CGT and CTOP is not feasible for intermediate COT because they are both sensitive to the mean of A-band ratio. The joint retrieval should, however, be possible for optically thin and thick clouds; the problem is to determine the limit between those cases as it could depend on cloud microphysics and/or illumination and viewing geometry.

\subsection{Impact of cloud microphysics}

To evaluate whether cloud microphysics could have an impact on available information content, we studied DOFs for different assumptions on the droplet effective radius, and no significant effects on the information content on CGT and CTOP were noticed (not shown). This is not surprising since the asymmetry factor $g$ is the relevant optical parameter that

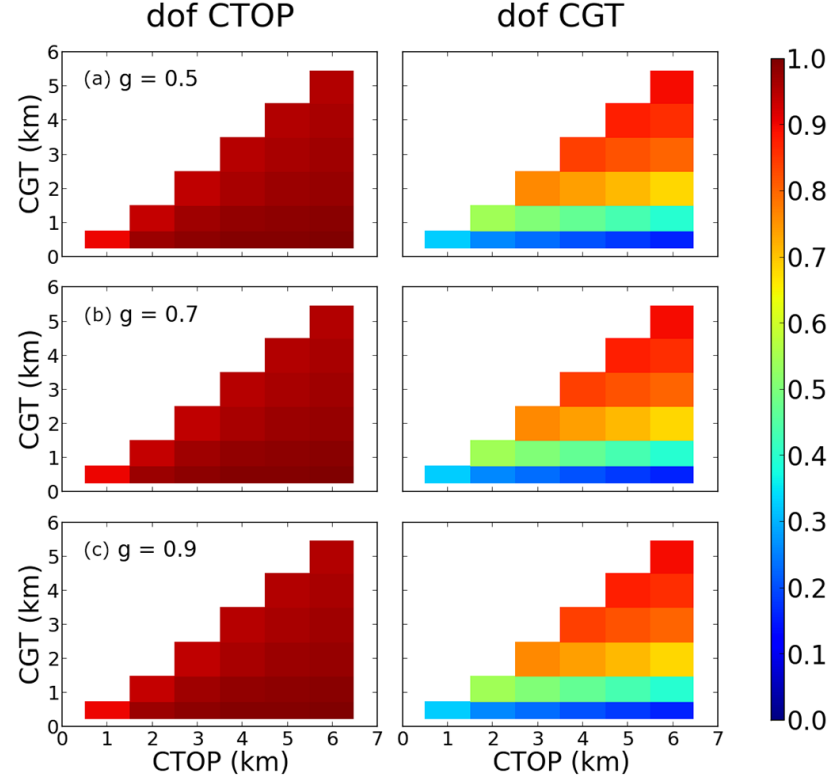

Figure 15. Information content of 3MI A-band with a COT of 16 for different asymmetry factors: (a) $g=0.5$, (b) $g=0.7$, (c) $g=0.9$. Partial DOFs for CTOP (left) and CGT (right).

links cloud microphysics to photon penetration within the cloud (Davis et al., 2009) and, for liquid droplets at least, it does not vary much at the A-band wavelength for the range of potential droplet sizes.

To further confirm this finding, we imposed larger variation and uncertainty on the asymmetry factor by using the Henyey-Greestein phase function for the cloud's scattering properties. Figure 15 represents the partial degrees of freedom on CTOP and CGT in the same case as Fig. 11 but for three different asymmetry factor values: $g=0.5,0.7$ and 0.9. In this case, the forward model was calculated with Henyey-Greenstein phase functions with $10 \%$ error on the non-retrieved parameter $g$. The value of $g=0.7$ (Fig. 15b) corresponds approximately to ice clouds, the value $g=0.9$ (Fig. 15c) to liquid clouds, and the value $g=0.5$ (Fig. 15a) represents an extreme lower value. Again those three cases do not show significant differences. We therefore conclude that the feasibility of retrieving CTOP and CGT will not be impacted by uncertainties about cloud microphysics as a priori knowledge about asymmetry factor can be provided to better than $10 \%$. Although the Henyey-Greenstein phase function provides a practical way to vary the asymmetry factor, one may question whether it can realistically represent ice clouds in our problem of interest. Similar results (not shown) are obtained when one uses realistic ice cloud phase function (IHM - Inhomogeneous Hexagonal Model, Labonnote et al. (2001)) and for higher cloud top altitudes. Figure 16 is the same as Fig. 14 but for ice clouds, with the IHM phase function model and high CTOP (from 7 to $13 \mathrm{~km}$ ). We notice that the degrees of freedom on CTOP is high for the 


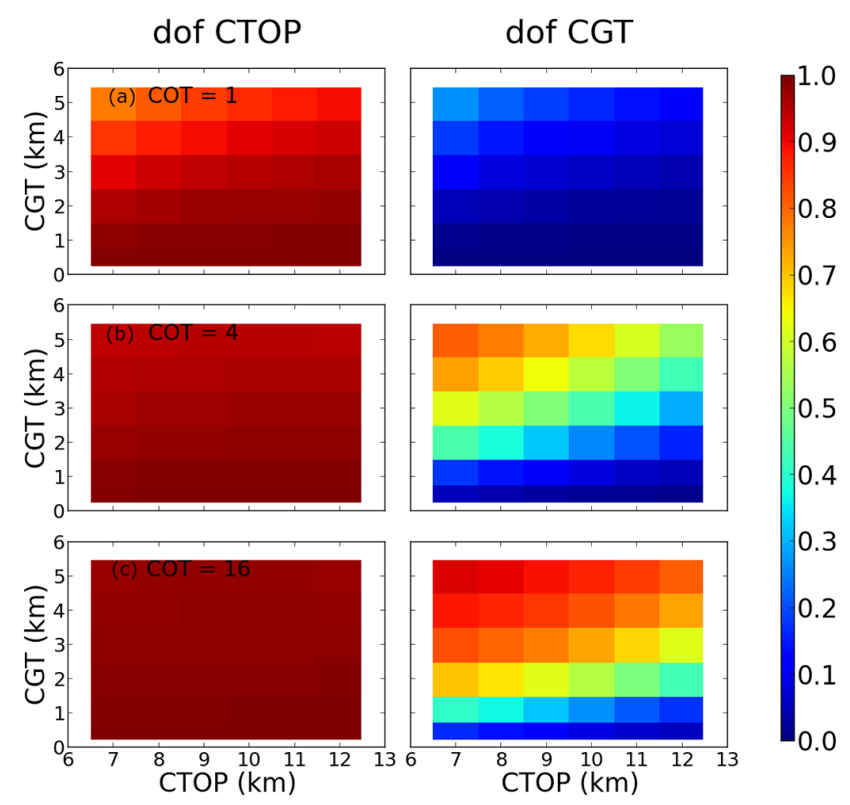

Figure 16. Information content of 3MI A-band for ice clouds (IHM model) for different COTs: (a) $\mathrm{COT}=1$, (b) $\mathrm{COT}=4$, (c) $\mathrm{COT}=16$. Partial DOFs for CTOP (left) and CGT (right).

three COT presented in this figure even if it decreases a little when COT decreases. As for liquid clouds (Fig. 14), the DOFs on CTOP increase with CTOP and decrease with CGT. Concerning CGT, the information on CGT is very high for $\mathrm{COT}=16$ and for CGT larger than $2 \mathrm{~km}$. It is consistent with liquid clouds results but, for ice cloud, the DOF decreases with COT and there is no more information on CGT for $\mathrm{COT}=1$. The retrieval of CTOP seems then to be possible for all COT for ice clouds. For CGT, it is not possible for low COT $(=1)$ and for CGT smaller than $2 \mathrm{~km}$.

Therefore the results of the information content presented in this paper can be confidently extended to different types of clouds such as ice clouds (at least for high optical thicknesses).

\subsection{Variations in solar and viewing geometry}

The viewing geometry resulting from angular sampling (AS) could be another important parameter. To restrict the number of angular configurations, we chose three different AS precomputed for 3MI orbits, as described in Fig. 2. We made the choice to obtain representative observational configurations. Indeed, with a SZA of $30^{\circ}$, AS/orbit 3 corresponds to measurements in the backward-scattering direction, AS/orbit 1 in the forward scattering direction and AS 2 between them. Figure 17 shows the results of information content for these geometries applied to the reference case. The information content provided by these three configurations is very similar. We only notice slightly less information for AS/orbit 2 and 3 that might be due to a smaller contribution of the mul-

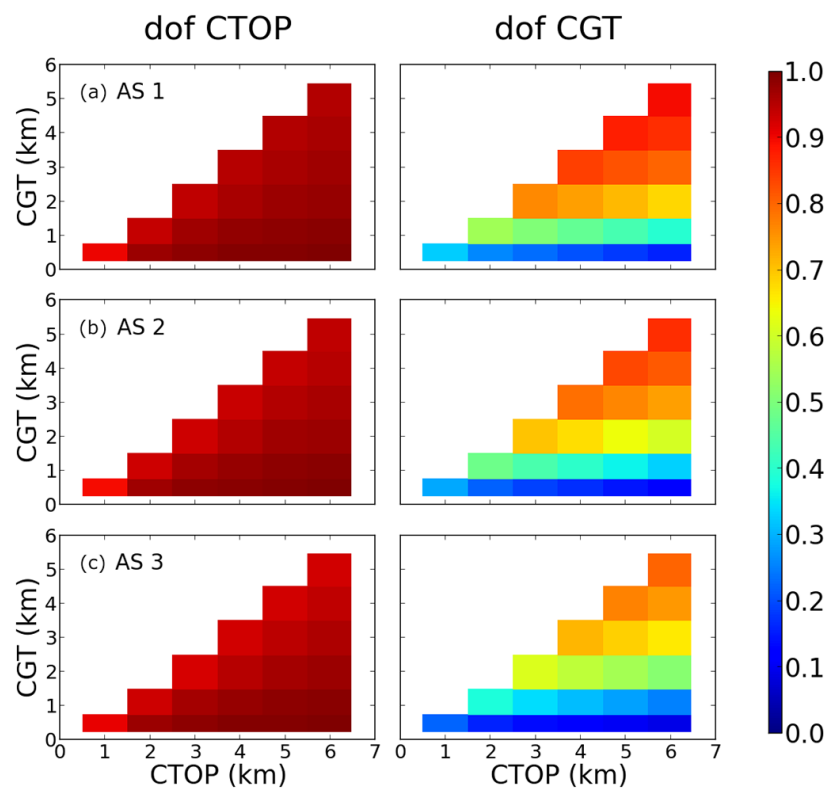

Figure 17. Information content of 3MI A-band with a COT of 16 for different orbits, hence the following angular samplings: (a) AS 1, (b) AS 2, (c) AS 3. Partial DOFs for CTOP (left) and CGT (right).

tiple scattering, hence reduced penetration of sunlight and photon path length into the cloud mass.

To gauge the effect of the SZA, we performed the same study but for two alternate settings, 0 and $60^{\circ}$, with the angular configuration in AS 1; see Fig. 18. As previously, the outcomes of cases $\mathrm{SZA}=30$ and $60^{\circ}$ are quite close, and $\mathrm{SZA}=0^{\circ}$ gives a little less information. This configuration corresponds to the closest configuration of the backwardscattering directions (see AS 3 in Fig. 17). This confirms that the information is somewhat weaker in the backwardscattering direction.

\subsection{Addition of a Lambertian surface with varying albedo}

We showed in Sect. 3.2 that adding a surface decreases the sensitivity of the A-band on CTOP and CGT. Figure 19 shows the information content on CTOP and CGT when considering a surface albedo of 0.2, typical of land (and assumed known to \pm 0.05 ) for the three different optical thicknesses studied before. This figure is to be compared with Fig. 14 for a black surface. First, for optically thick clouds (COT $=16$, Fig. 19c) the information content does not change compared to Fig. 14. The cloud is too opaque and such surface has no effect on the A-band ratio. The case of COT $=4$ is hardly affected by the addition of the surface either. However, for optically thin $(\mathrm{COT}=1)$ clouds, the impact of the surface is important. Information on both CTOP and CGT decreases. It was already noticed qualitatively in Sect. 3 (Fig. 7a) that the sensitivities on CTOP and CGT are lower above brighter surfaces. By taking into account this decrease of sensitivity and 


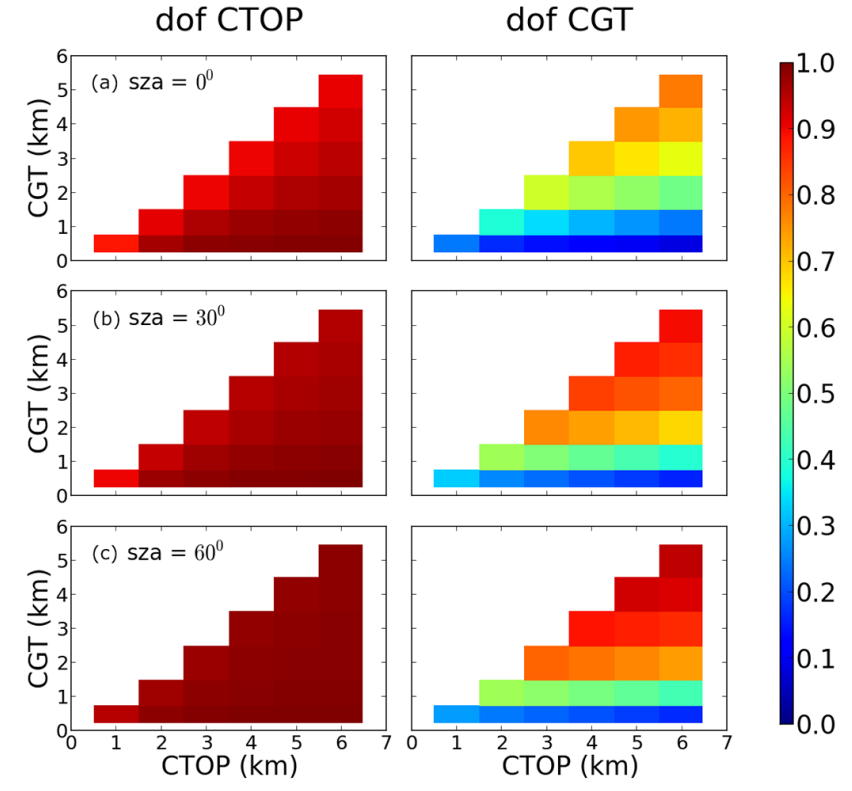

Figure 18. Information content of $3 \mathrm{MI}$ A-band with a COT of 16 for different illumination angles: (a) $\mathrm{SZA}=0^{\circ}$, (b) $\mathrm{SZA}=30^{\circ}$, (c) $\mathrm{SZA}=60^{\circ}$. Partial DOFs for CTOP (left) and CGT (right).

the forward model errors, information on CGT will become very low and the retrieval of CGT impossible for low values of COT. Figure 20 presents the information content for the same cases but with a surface albedo of 0.8 , representative of fresh snow or ice. As before, information for $\mathrm{COT}=1$ becomes too low. However, in comparison with Fig. 19, and contrary to what we expected looking at Fig. 9, the high information content for optically thick clouds $(\mathrm{COT}=16$, Fig. 20c) is not dramatically affected by such bright surfaces. For COT $=4$, the information on CGT interestingly increases compared to a black surface where both CGT and CTOP were sensitive to the average ratio, but not the standard deviation (cf. Fig. 6). We attribute this increase in sensitivity to cloud properties to the fact that clouds of intermediate optical depth are highly transmissive in diffuse light, and much of this transmitted light is retransmitted by the cloud after surface reflection, accumulating more path length in the dioxygen.

For bright surfaces, Figs. 9 and 10 show that CTOP is rather sensitive to the angular standard deviation and CGT rather to the mean of the A-band ratio. We have thus independent information on CTOP and CGT. For optically thin clouds $(\mathrm{COT}=1)$, for some cases of CTOP and CGT, the information is higher and for some others it is lower than less bright surfaces. This case is very non-linear due to multiple reflections between surface and cloud. The A-band ratio will depend strongly on surface albedo, COT and absorption below the cloud which depends on the cloud base altitude. We can also notice that information on CGT is not sufficient in most cases, contrary to CTOP.

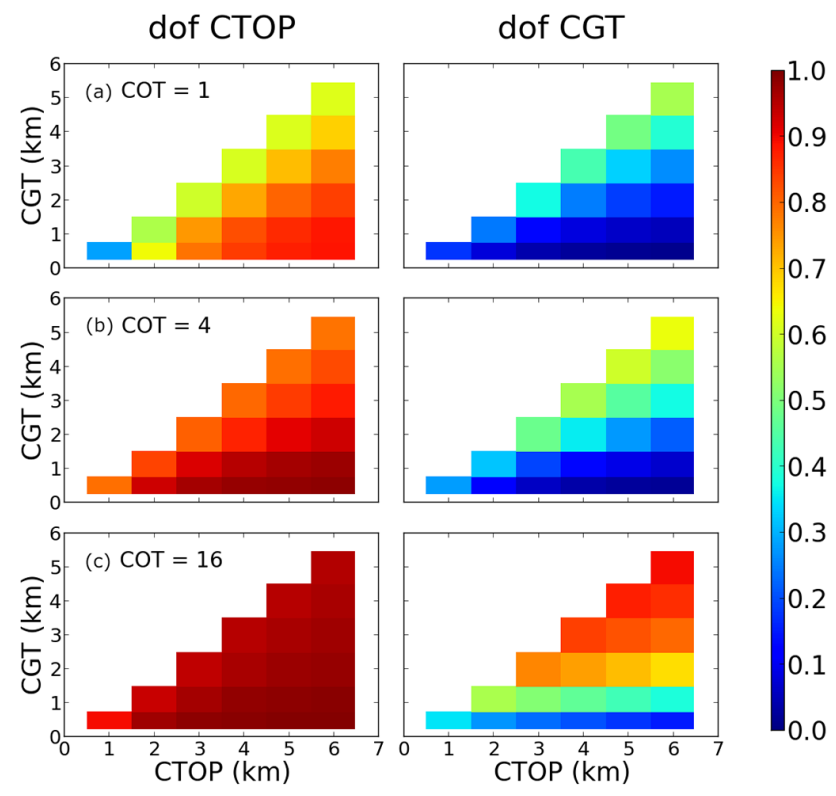

Figure 19. Same as Fig. 14 with an albedo of 0.2.

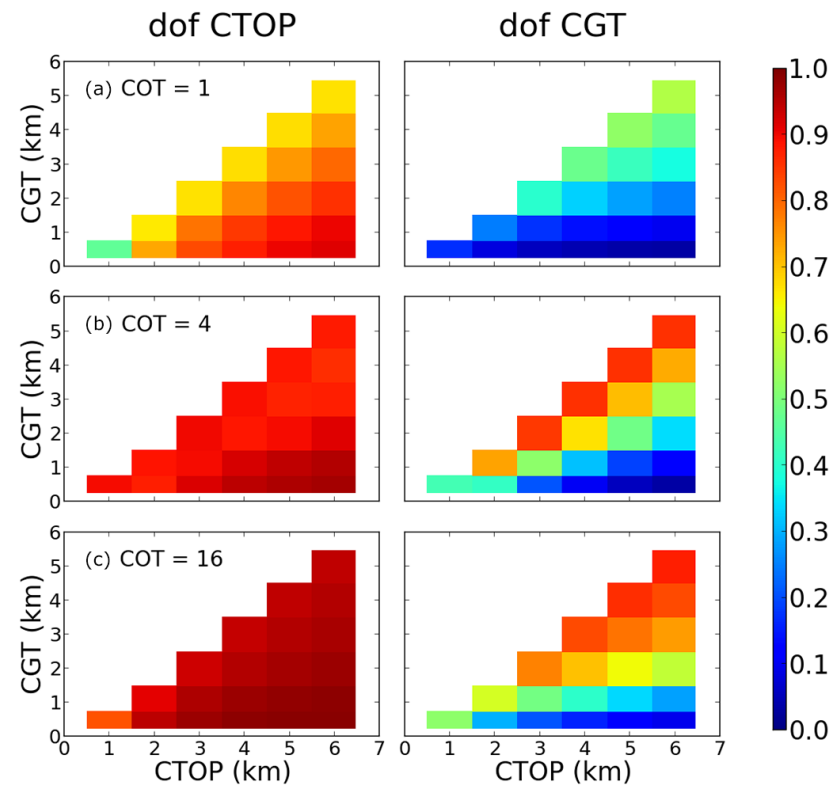

Figure 20. Same as Figs. 14 and 19 with an albedo of 0.8. There is an interesting increase in information content for the moderately opaque $(\mathrm{COT}=4)$ clouds when they are relatively thick, e.g. CGT $\geq \max \{2, \mathrm{CTOP}-2\} \mathrm{km}$.

To summarize, the retrieval of CTOP over bright surfaces is feasible regardless of the COT and albedo, noting that both properties need to known, although not perfectly. Cloud top altitude can be determined here because, depending on the optical thickness, a significant part of the radiation is reflected near the cloud top and is more or less absorbed depending on its altitude. While this part of the cloud-reflected 
radiation is sufficient to impact the total signal, information only on cloud top altitude can be retrieved even above a bright surface. However, this finding should be mitigated by the fact that we assume that the cloud optical thickness is known within $10 \%$ accuracy, which might be difficult to achieve over very bright surfaces. This is also the case for CGT along with CTOP for optically thick clouds. For optically thin clouds, on the other hand, retrieving CGT seems to remain challenging using the anticipated $3 \mathrm{MI}$ spectral configuration.

\section{Conclusions}

The main goal of the present work has been to study the $\mathrm{O}_{2}$ A-band in terms of information content for multi-angular two-channel measurements provided by different future instruments, specifically 3MI and MSPI. Although earlier studies already revealed the sensitivity of the A-band to the cloud top altitude and geometrical thickness, they did not always account for measurement noise and errors due to uncertainties in the non-retrieved parameters and were limited to thick clouds above black surfaces. In this study, we consider optically thick clouds as well as optically intermediate and thin clouds. In addition, we look at the information content above Lambertian surfaces with moderate and high albedo. The main results are as follows.

1. MSPI's configuration seems to be more appropriate and the use of an external non-absorbing band seems more efficient than a broad spectral channel covering the Aband and some neighbouring continuum wavelengths.

2. Information on CTOP is high in almost all studied cases and this parameter seems to be retrievable using an optimal estimation scheme.

3. The retrieval of CGT seems to pose problems in some cases. Over a black surface, information could be too low for CGT smaller than 1-2 km. Over a brighter surface, this information is decreased for most of cases, but not for optically thick clouds.

Interestingly, sensitivity to CGT increases for relatively thick (CGT $\gtrsim \max \{2, \mathrm{CTOP}-2\} \mathrm{km}$ ) clouds of intermediate optical thickness $(\mathrm{COT} \approx 4)$ when they occur over the brightest surfaces, due to the extra path length cumulated in the cloud after surface reflection.

This study shows the possibility of retrieving cloud top and cloud geometrical thickness. However, in order to develop an operational algorithm, precautions should be taken in case of heterogeneous cloud or when aerosols lay above clouds as both will modify the photon path length, hence modifying the absorption of the radiation. This study is restricted to homogeneous plane-parallel clouds, but cloud inhomogeneity and three-dimensional effects are known to modify significantly the multi-angular measurement but also the photon path length. We have shown that the angular distribution of observed $\mathrm{O}_{2}$ A-band absorption carries information on cloud geometrical thickness. Though the absolute values of radiances are known to be directly impacted by threedimensional effects, it remains to be established to what extent this modifies the relative angular distribution of observed $\mathrm{O}_{2}$ A-band absorption as derived from two-band ratios. In this respect, future studies will investigate cases of heterogeneous cloud covers in order to estimate the effects of cloud inhomogeneity on the information content evaluated here as well as the implications on the retrieval of both cloud top height and geometrical thickness.

This study aimed to determine the capabilities of multiangle A-band measurements, but other spectral regions could be useful to retrieve CTOP and CGT. For instance, polarized radiances in the deep blue are sensitive to CTOP (Goloub et al., 1994). It shall be noted that other planned or already launched missions carrying sensors that provide measurements in the $\mathrm{O}_{2}$ A- and B-bands could still benefit from our analysis. As an example, we can take the Japanese Second Generation Global Imager of the Global Change Observation Mission - Climate (SGLI/GCOM-C) and the Earth Polychromatic Imaging Camera on the DSCOVR spacecraft (EPIC/DSCOVR) that observes the sunlit Earth from the Lagrange "L1" point. Additionally, but outside the of scope of the present study, A-band measurements at high spectral resolution from OCO-2 or GOSAT, even at a single-view angle, could also be used to retrieve similar cloud information with the additional limitation of coarse spatial resolution leading to larger uncertainties in the forward model.

\section{Data availability}

All simulations for this study have been performed using the ARTDECO simulation package. ARTDECO (Atmospheric Radiative Transfer Database for Earth Climate Observation) is a numerical tool that gathers several models and data for the simulation of Earth atmosphere radiances and radiative fluxes as observed with passive sensors (hyperspectral excluded) in the UV to thermal IR range. The full software suite can be obtained through the ICARE Data and Services Center (2016) at http://www.icare.univ-lille1.fr/projects/artdeco. 


\section{Appendix A: The optimal estimation approach to information content analysis}

In the Rodgers (2000) formalism for the optimal estimation of model parameters from observations, measurements are represented by the "data" $m$-vector $\boldsymbol{y}$ (in this study containing the multi-angle suite of A-band ratios, e.g. $m=9$ and 15 for MSPI and 3MI) and the retrieved parameters are contained in the "state" $n$-vector $\boldsymbol{x}$ (here containing CTOP and CGT, i.e. $n=2$ ). The corresponding information content theory uses Gaussian probability functions (PDFs) to relate, through Bayes' theorem, the measurement space and the state space. Since the PDFs are assumed to follow multi-variate Gaussian distributions, they are defined by their means and variance-covariance matrices:

- $\mathbf{S}_{a}$ for the PDF of the a priori state vector representing our "knowledge" of the state vector before any measurements are made;

- $\mathbf{S}_{x}$ for the PDF of the a posteriori state vector representing our "knowledge" of the state vector accounting for the measurements;

- $\mathbf{S}_{\epsilon}$ represents the variance-covariance matrix of the measurement and the forward model errors.

We first need to define the forward model variancecovariance matrix, particularly the errors, due to the nonretrieved parameters we use in the forward model. $\mathbf{S}_{b}$ will be the variance-covariance matrix of those non-retrieved parameters (assumed diagonal). The variance-covariance matrix associated to the model errors $\mathbf{S}_{f}$ is therefore computed as

$\mathbf{S}_{f}=\mathbf{K}_{b} \mathbf{S}_{b} \mathbf{K}_{b}^{\mathrm{T}}$,

with $\mathbf{K}_{b}$ being the Jacobian of the non-retrieved parameters, which represents the sensitivity of the forward model to those non-retrieved parameters, and superscript " $T$ " meaning transpose.

We can then compute the variance-covariance matrix $\mathbf{S}_{\epsilon}$ of the measurements and forward model errors, $\mathbf{S}_{y}$ and $\mathbf{S}_{f}$ :

$\mathbf{S}_{\epsilon}=\mathbf{S}_{y}+\mathbf{S}_{f}$.

The a posteriori variance-covariance matrix $\mathbf{S}_{x}$, with the diagonal elements representing the uncertainties on the best retrieval, is expressed as

$\mathbf{S}_{x}=\left(\mathbf{S}_{a}^{-1}+\mathbf{K}^{\mathrm{T}} \mathbf{S}_{\epsilon}^{-1} \mathbf{K}\right)^{-1}$

The term $\mathbf{K}$ represents the kernel (or Jacobian) matrix, which describes the sensitivity of the forward model to each parameter to be retrieved. A posteriori retrieval errors for the $i$ th parameter are

$\sigma_{i}=\sqrt{\mathbf{S}_{x, i i}}$.
The notion of information content was introduced by Shannon (1948). They developed this theory by establishing an analogy between the information carried by a signal and the entropy of its PDF. Later, Rodgers (2000) applied this idea to his formalism. He defined the information content $H_{s}$ as the difference between the a priori and a posteriori entropies of the respective Gaussian PDFs defined by the matrices $\mathbf{S}_{a}$ and $\mathbf{S}_{x}$ :

$H_{s}=\frac{1}{2} \log _{2}\left(\left\|\mathbf{S}_{x} S_{a}^{-1}\right\|\right)$.

$H_{s}$ thus quantifies the reduction of the number of possible states from the a priori state space to the best-estimate a posteriori state space. Rodgers describes another way of quantifying this volume reduction, by using the averaging kernel matrix:

$A=\frac{\partial \hat{\boldsymbol{x}}}{\partial \boldsymbol{x}}$,

with $\hat{\boldsymbol{x}}$ representing the best estimate of the state vector and $\boldsymbol{x}$ the true state vector. $A$ is therefore the sensitivity of the retrieval to the true state. It is a simple function of the a priori and a posteriori volumes:

$A=\mathbf{I}_{n}-\mathbf{S}_{x} \mathbf{S}_{a}^{-1}$,

where $\mathbf{I}_{n}$ is the $n \times n$ identity matrix. From Eqs. (A5) and (A7), we can express the information content $H_{S}$ as follows:

$H_{s}=\frac{1}{2} \log _{2}\left(\left\|\mathbf{I}_{n}-A\right\|\right)$.

We can also express the total number of degrees of freedom $d_{s}$ as a function of the averaging kernel matrix. The degree of freedom is a measure of the number of independent pieces of information contained in the observing system. It is expressed as

$d_{s}=\operatorname{Tr}(A)$.

Note that its maximum value is equal to the dimension $n$ of the state vector. We can also get the partial degree of freedom of the $i$ th parameter attached to each element of the state vector of $\boldsymbol{x}$ :

$d_{s, i}=A_{i i}$.

It represents the information brought by the measurements for the parameter $x_{i}(i=1, \ldots, n)$.

This partial degree of freedom is a very practical tool for quantifying information content. Indeed, $d_{s, i}$ lies between 0 and 1 , with a low value representing a low information and conversely when it approaches unity. Partial degree of freedom is therefore what we use throughout our study, with the a posteriori variance-covariance matrix $\mathbf{S}_{x}$ in Eq. (A3), to study the information content of our measurements, namely, multi-angle $\mathrm{O}_{2}$ A-band in/out ratios. Note, from Eq. (A4) on the one hand and from Eqs. (A7) and (A10) on the other hand, that $d_{s, i}$ increases when $\sigma_{i}$ decreases. 
Acknowledgements. The authors are grateful for financial support from CNES as well as NASA's SMD/ESD (several programmes managed by H. Maring, K. Jucks and R. Eckman). They also thank Dave Diner, Jay Herman and Yuequi Yang for fruitful discussions. Guillaume Merlin is supported by a PhD grad from CNES and Région Nord-Pas de Calais.

Edited by: B. Kahn

Reviewed by: three anonymous referees

\section{References}

Asano, S., Shiobara, M., and Uchiyama, A.: Estimation of cloud physical parameters from airborne solar spectral reflectance measurements for stratocumulus clouds, J. Atmos. Sci., 52, 35563576, 1995.

Bréon, F.-M. and Doutriaux-Boucher, M.: A comparison of cloud droplet radii measured from space, IEEE T. Geosci. Remote, 43, 1796-1805, doi:10.1109/TGRS.2005.852838, 2005.

Cooper, S. J., L'Ecuyer, T. S., Gabriel, P., Baran, A. J., and Stephens, G. L.: Objective assessment of the information content of visible and infrared radiance measurements for cloud microphysical property retrievals over the global oceans. Part II: Ice clouds, J. Appl. Meteorol. Clim., 45, 42-62, doi:10.1175/JAM2327.1, 2006.

Davis, A., Polonsky, I., and Marshak, A.: Space-time Green functions for diffusive radiation transport, in: application to active and passive cloud probing, in: Light Scattering Reviews, edited by: Kokhanovsky, A., vol. 4, 169-292, Springer-Praxis, Heidelberg, Germany, 2009.

Davis, A. B. and Marshak, A.: Solar radiation transport in the cloudy atmosphere: a 3D perspective on observations and climate impacts, Rep. Prog. Phys., 73, 26801, doi:10.1088/00344885/73/2/026801, 2010.

de Beek, R., Vountas, M., Rozanov, V. V., Richter, a., and Burrows, J. P.: The ring effect in the cloudy atmosphere, Geophys. Res. Lett., 28, 721-724, doi:10.1029/2000GL012240, 2001.

de Haan, J., Bosma, P., and Hovenier, J.: The adding method for multiple scattering calculations of polarized light, Astron. Astrophys., 183, 371-391, 1987.

Desmons, M., Ferlay, N., Parol, F., Mcharek, L., and Vanbauce, C.: Improved information about the vertical location and extent of monolayer clouds from POLDER3 measurements in the oxygen A-band, Atmos. Meas. Tech., 6, 2221-2238, doi:10.5194/amt-62221-2013, 2013.

Di Girolamo, L., Liang, L., and Platnick, S.: A global view of one-dimensional solar radiative transfer through oceanic water clouds, Geophys. Res. Lett., 37, 1-5, doi:10.1029/2010GL044094, 2010.

Diner, D. J., Davis, A., Hancock, B., Geier, S., Rheingans, B., Jovanovic, V., Bull, M., Rider, D. M., Chipman, R. A., Mahler, A.-B., and McClain, S. C.: First results from a dual photoelasticmodulator-based polarimetric camera, Appl. Optics, 49, 29292946, 2010.

Diner, D. J., Xu, F., Garay, M. J., Martonchik, J. V., Rheingans, B. E., Geier, S., Davis, A., Hancock, B. R., Jovanovic, V. M., Bull, M. A., Capraro, K., Chipman, R. A., and McClain, S. C.: The Airborne Multiangle SpectroPolarimetric Imager (AirMSPI): a new tool for aerosol and cloud remote sensing, Atmos. Meas. Tech., 6, 2007-2025, doi:10.5194/amt-6-2007-2013, 2013.

Ferlay, N., Thieuleux, F., Cornet, C., Davis, A. B., Dubuisson, P., Ducos, F., Parol, F., Riédi, J., and Vanbauce, C.: Toward New Inferences about Cloud Structures from Multidirectional Measurements in the Oxygen A Band: Middleof-Cloud Pressure and Cloud Geometrical Thickness from POLDER-3/PARASOL, J. Appl. Meteorol. Clim., 49, 2492 2507, doi:10.1175/2010JAMC2550.1, 2010.

Fougnie, B.: Improvement of the PARASOL Radiometric In-Flight Calibration Based on Synergy Between Various Methods Using Natural Targets, IEEE T. Geosci. Remote, 54, 2140-2152, 2016.

Goloub, P., Deuze, J. L., Herman, M., and Fouquart, Y.: Analysis of the POLDER polarization measurements performed over cloud covers, IEEE T. Geosci. Remote, 32, 78-88, doi:10.1109/36.285191, 1994.

Heidinger, A. K. and Stephens, G. L.: Molecular Line Absorption in a Scattering Atmosphere. Part II: Application to Remote Sensing in the $\mathrm{O}_{2}$ A band, J. Atmos. Sci., 57, 1615-1634, doi:10.1175/1520-0469(2000)057<1615:MLAIAS>2.0.CO;2, 2000.

Heidinger, A. K. and Stephens, G. L.: Molecular Line Absorption in a Scattering Atmosphere. Part III: Pathlength Characteristics and Effects of Spatially Heterogeneous Clouds, J. Atmos. Sci., 59, 1641-1654, doi:10.1175/15200469(2002)059<1641:MLAIAS>2.0.CO;2, 2002.

ICARE Data and Services Center: Atmospheric Radiative Transfer Database for Earth Climate Observation (ARTDECO) tool, available at: http://www.icare.univ-lille1.fr/projects/artdeco, last access: 10 October 2016.

Johansson, E., Devasthale, A., L'Ecuyer, T., Ekman, A. M. L., and Tjernström, M.: The vertical structure of cloud radiative heating over the Indian subcontinent during summer monsoon, Atmos. Chem. Phys., 15, 11557-11570, doi:10.5194/acp-1511557-2015, 2015.

Joiner, J. and Bhartia, P. K.: The determination of cloud pressures from rotational Raman scattering in satellite backscatter ultraviolet measurements, J. Geophys. Res., 100, 23019-23026, doi:10.1029/95JD02675, 1995.

King, M. D., Kaufman, Y. J., Menzel, W. P., and Tanre, D.: Remote sensing of cloud, aerosol, and water vapor properties from the Moderate Resolution Imaging Spectrometer (MODIS), IEEE T. Geosci. Remote, 30, 2-27, 1992.

King, N. J. and Vaughan, G.: Using passive remote sensing to retrieve the vertical variation of cloud droplet size in marine stratocumulus: An assessment of information content and the potential for improved retrievals from hyperspectral measurements, J. Geophys. Res., 117, D15206, doi:10.1029/2012JD017896, 2012.

Knibbe, W. J. J., De Haan, J. F., Hovenier, J. W., Stam, D. M., Koelemeijer, R. B. A., and Stammes, P.: Deriving terrestrial cloud top pressure from photopolarimetry of reflected light, J. Quant. Spectrosc. Ra., 64, 173-199, doi:10.1016/S0022-4073(98)00135-6, 2000.

Kratz, D. P.: The correlated $k$-distribution technique as applied to the AVHRR channels, J. Quant. Spectrosc. Ra., 53, 501-517, doi:10.1016/0022-4073(95)90050-0, 1995.

Kuji, M. and Nakajima, T.: Retrieval of Cloud Geometrical Parameters Using Remote Sensing Data, 11th Conf. on Cloud Physics, 4150, JP1.7, 2002. 
Labonnote, L. C., Brogniez, G., Buriez, J. C., Doutriaux Boucher, M., Gayet, J. F., and Macke, A.: Polarized light scattering by inhomogeneous hexagonal monocrystals: Validation with ADEOS-POLDER measurements, J. Geophys. Res.-Atmos., 106, 12139-12153, doi:10.1029/2000JD900642, 2001.

L'Ecuyer, T. S., Wood, N. B., Haladay, T., Stephens, G. L., and Stackhouse, P. W.: Impact of clouds on atmospheric heating based on the R04 CloudSat fluxes and heating rates data set, J. Geophys. Res.-Atmos., 114, 1-15, doi:10.1029/2008JD009951, 2009.

Liang, L. and Girolamo, L. D.: A global analysis on the view-angle dependence of plane-parallel oceanic liquid water cloud optical thickness using data synergy from MISR and MODIS, J. Geophys. Res.-Atmos., 118, 2389-2403, doi:10.1029/2012JD018201, 2013.

Loeb, N. and Coakley Jr, J.: Inference of marine stratus cloud optical depths from satellite measurements: Does 1D theory apply?, J. Climate, 11, 215-233, doi:10.1175/15200442(1998)011<0215:IOMSCO>2.0.CO;2, 1998.

Loyola, D. G., Thomas, W., Livschitz, Y., Ruppert, T., Albert, P., and Hollmann, R.: Cloud properties derived from GOME/ERS-2 backscatter data for trace gas retrieval, IEEE T. Geosci. Remote, 45, 2747-2758, doi:10.1109/TGRS.2007.901043, 2007.

Mace, G. G., Zhang, Q., Vaughan, M., Marchand, R., Stephens, G., Trepte, C., and Winker, D.: A description of hydrometeor layer occurrence statistics derived from the first year of merged Cloudsat and CALIPSO data, Journal of Geophysical Research, 114, D00A26, doi:10.1029/2007JD009755, 2009.

Moroney, C., Davies, R., and Muller, J.-P.: Operational retrieval of cloud-top heights using MISR data, IEEE T. Geosci. Remote, 40, 1532-1540, doi:10.1109/TGRS.2002.801150, 2002.

Ohring, G. and Adler, S.: Some experiments with zonally averaged climate model, J. Atmos. Sci., 35, 186-205, 1978.

Platnick, S., King, M. D., Ackerman, S., Menzel, W. P., Baum, B., Riedi, J. C., and Frey, R.: The MODIS cloud products: Algorithms and examples from Terra, IEEE T. Geosci. Remote, 41, 459-473, doi:10.1109/TGRS.2002.808301, 2003.

Preusker, R. and Lindstrot, R.: Remote sensing of cloud-top pressure using moderately resolved measurements within the oxygen A band - A sensitivity study, J. Appl. Meteorol. Clim., 48, 15621574, doi:10.1175/2009JAMC2074.1, 2009.

Ramon, D., Richard, S., and Dubuisson, P.: MERIS in-flight spectral calibration in $\mathrm{O}_{2}$ absorption using surface pressure retrieval, Proceeding of SPIE, 4891, 505-514, 2003.

Riedi, J., Marchant, B., Platnick, S., Baum, B. A., Thieuleux, F., Oudard, C., Parol, F., Nicolas, J.-M., and Dubuisson, P.: Cloud thermodynamic phase inferred from merged POLDER and MODIS data, Atmos. Chem. Phys., 10, 11851-11865, doi:10.5194/acp-10-11851-2010, 2010.

Rodgers, C. D.: Information content and optimisation of high spectral resolution remote measurements, Adv. Space Res., 21, 361367, 1998 .

Rodgers, C. D.: Inverse Methods for Atmospheric Sounding: Theory and Practice, World Scientific, Singapore, 2000.

Rossow, W. B. and Schiffer, R. A.: ISCCP Cloud Data Products, B. Am. Meteorol. Soc., 72, 2-20, doi:10.1175/15200477(1991)072<0002:ICDP>2.0.CO;2, 1991.

Rozanov, V. V. and Kokhanovsky, A. A.: Semianalytical cloud retrieval algorithm as applied to the cloud top altitude and the cloud geometrical thickness determination from top-of-atmosphere reflectance measurements in the oxygen A band, J. Geophys. Res.Atmos., 109, D05202, doi:10.1029/2003JD004104, 2004.

Schuessler, O., Loyola, D., Doicu, A., and Spurr, R.: Information content in the oxygen-band for the retrieval of macrophysical cloud parameters, IEEE T. Geosci. Remote, 52, 3246-3255, 2014.

Seiz, G., Tjemkes, S., and Watts, P.: Multiview Cloud-Top Height and Wind Retrieval with Photogrammetric Methods: Application to Meteosat-8 HRV Observations, J. Appl. Meteorol. Clim., 46, 1182-1195, doi:10.1175/JAM2532.1, 2007.

Shannon, C. E.: A mathematical theory of communication, Bell Syst. Tech. J., 27, 379-423, 1948.

Sourdeval, O., -Labonnote, L. C., Brogniez, G., Jourdan, O., Pelon, J., and Garnier, A.: A variational approach for retrieving ice cloud properties from infrared measurements: application in the context of two IIR validation campaigns, Atmos. Chem. Phys., 13, 8229-8244, doi:10.5194/acp-13-8229-2013, 2013.

Sourdeval, O., C.-Labonnote, L., Baran, A. J., and Brogniez, G.: A methodology for simultaneous retrieval of ice and liquid water cloud properties. Part I: Information content and case study, Q. J. Roy. Meteor. Soc., 141, 870-882, doi:10.1002/qj.2405, 2014.

Stocker, T., Qin, D., Plattner, G.-K., Tignor, M., Allen, S., Boschung, J., Nauels, A., Xia, Y., Bex, V., and Midgley, P.: IPCC 2013: Climate Change 2013: The Physical Science Basis. Contribution of Working Group I to the Fifth Assessment Report of the Intergovernmantal Panel on climate Change, Tech. rep., Cambridge University Press, Cambridge, UK and New York, NY, USA, 2013.

Várnai, T. and Marshak, A.: View angle dependence of cloud optical thicknesses retrieved by Moderate Resolution Imaging Spectroradiometer (MODIS), J. Geophys. Res.-Atmos., 112, D06203, doi:10.1029/2005JD006912, 2007.

Winker, D. M. and Trepte, C. R.: Laminar cirrus observed near the tropical tropopause by LITE, Geophys. Res. Lett., 25, 33513354, doi:10.1029/98GL01292, 1998.

Winker, D. M., Hunt, W. H., and McGill, M. J.: Initial performance assessment of CALIOP, Geophys. Res. Lett., 34, L19803, doi:10.1029/2007GL030135, 2007

Wu, D. L., Ackerman, S. a., Davies, R., Diner, D. J., Garay, M. J., Kahn, B. H., Maddux, B. C., Moroney, C. M., Stephens, G. L., Veefkind, J. P., and Vaughan, M. A.: Vertical distributions and relationships of cloud occurrence frequency as observed by MISR, AIRS, MODIS, OMI, CALIPSO, and CloudSat, Geophys. Res Lett., 36, L09821, doi:10.1029/2009GL037464, 2009.

Yamamoto, G. and Wark, D. Q.: Discussion of the letter by R. A. Hanel, "Determination of cloud altitude from a satellite", J. Geophys. Res., 66, 3596, doi:10.1029/JZ066i010p03596, 1961.

Yang, Y., Marshak, A., Mao, J., Lyapustin, A., and Herman, J.: A method of retrieving cloud top height and cloud geometrical thickness with oxygen $\mathrm{A}$ and $\mathrm{B}$ bands for the Deep Space Climate Observatory (DSCOVR) mission: Radiative transfer simulations, J. Quant. Spectrosc. Ra., 122, 141-149, doi:10.1016/j.jqsrt.2012.09.017, 2013.

Zhang, Z., Yang, P., Kattawar, G., Riedi, J., -Labonnote, L. C., Baum, B. A., Platnick, S., and Huang, H.-L.: Influence of ice particle model on satellite ice cloud retrieval: lessons learned from MODIS and POLDER cloud product comparison, Atmos. Chem. Phys., 9, 7115-7129, doi:10.5194/acp-9-7115-2009, 2009. 